\title{
Presence of dust with a UV bump in massive, star-forming galaxies at $1<z<2.5^{\star}$
}

\author{
S. Noll, D. Pierini, M. Pannella, and S. Savaglio
}

Max-Planck-Institut für extraterrestrische Physik, Giessenbachstr., 85748 Garching, Germany

e-mail: [snoll; dpierini ; maurilio; savaglio] @mpe.mpg.de

Received 8 January 2007 / Accepted 4 July 2007

\section{ABSTRACT}

\begin{abstract}
Aims. Fundamental properties of the extinction curve, like the slope in the rest-frame ultraviolet (UV) and the presence/absence of a broad absorption excess centred at $2175 \AA$ (the UV bump), are investigated for a sample of 108 massive, star-forming galaxies at $1<z<2.5$, selected from the FDF Spectroscopic Survey, the K20 survey, and the GDDS.

Methods. These characteristics are constrained from a parametric description of the UV spectral energy distribution (SED) of a galaxy, as enforced by combined stellar population and radiative transfer models for different geometries, dust/stars configurations, and dust properties.

Results. In at least one third of the sample, there is a robust evidence for extinction curves with at least a moderate UV bump. The presence of the carriers of the UV bump is more evident in galaxies with UV SEDs suffering from heavy reddening. We interpret these results as follows. The sample objects possess different mixtures of dust grains and molecules producing extinction curves in between the average ones of the Small and Large Magellanic Cloud, where the UV bump is absent or modest, respectively. Most of the dust embeds the UV-emitting stellar populations or is distributed out of the galaxy mid-plane. Alternatively, even dust with a pronounced UV bump, as for the average Milky-Way extinction curve, can be present and distributed in the galaxy mid-plane. In this case, variations of the continuum scattering albedo with wavelength or an age-dependent extinction are not sufficient to explain the previous trend with reddening. Hence, additional extraplanar dust has to be invoked. The data suggest that the carriers of the UV bump are associated with intermediate-age stellar populations, while they survive in the harshest UV-radiation fields owing to dust self-shielding.

Conclusions. The existence of different extinction curves implies that different patterns of evolution and reprocessing of dust exist at high redshift. Ignoring this may produce a non-negligible uncertainty on the star-formation rate estimated from the rest-frame UV.
\end{abstract}

Key words. galaxies: high-redshift - galaxies: starburst - galaxies: ISM - ISM: dust, extinction - ultraviolet: galaxies

\section{Introduction}

Extinction occurs whenever electromagnetic radiation propagates through a medium containing a mixture of dust grains and molecules. The dependence of absorption and scattering by dust from the wavelength of a photon is described by the extinction curve, which depends on the physical and chemical properties of dust grains and molecules (Whittet 2003). On a galaxy scale, the propagation of electromagnetic radiation through a dusty medium is described by the attenuation function. This is the combination of the extinction curve with the geometry of the system, in which a substantial fraction of the scattered light is returned to the observer's line of sight.

The direct determination of the extinction curve from observations is limited to our own Galaxy, the Magellanic Clouds, and M 31 (e.g., Fitzpatrick 2004; Clayton 2004). The most striking differences among the typical extinction curves of the Milky Way (MW) and the Large and Small Magellanic Clouds (LMC and SMC, respectively) occur in the UV domain, where small dust grains and molecules absorb and scatter with the highest efficiency. From the MW to the SMC, the presence of a broad absorption excess centred at $2175 \AA$ (the UV bump, see Witt \& Lillie 1973 and references therein) vanishes (almost) completely, while the slope of the extinction curve in the far-UV becomes steeper.

$\star$ Table 1 is only available in electronic form at http: //www . aanda.org
Multiple carriers (i.e., organic carbon and amorphous silicates) were suggested to explain the enigmatic, invariant central wavelength and variable bandwidth of the $2175 \AA$ feature (Bradley et al. 2005 and references therein). The ingredients of interstellar dust that originate the UV bump and the far-UV rise are in broadly similar proportions in the MW and LMC, but dust seems to be more radically different in the SMC (Whittet 2003). Differences of the same relevance exist within our own Galaxy for different sight lines and towards different environments, from dense molecular clouds to the diffuse interstellar medium (ISM; see Fitzpatrick 2004). They must reflect the extreme sensitivity of the small-sized dust components to the local chemical enrichment and energy budget (in terms of radiation field and shocks), as well as their selective removal from the size distribution owing to a number of physical processes (see Whittet 2003).

Nearby starburst galaxies have an extinction curve that seems to lack a $2175 \AA$ bump, as the SMC curve, and to have a steep far-UV rise, intermediate between the MW and SMC curves (Gordon et al. 1997). This extinction curve of starbursts was constrained from the modelling of the observed UV/optical broad-band colours. An alternative interpretation of the empirical attenuation law in starbursts (Calzetti et al. 1994) invokes a turbulent interstellar medium (Fischera et al. 2003). However, it adopts a foreground screen for the distribution of MW dust that is reminiscent of the dust/stars configuration of the Gordon et al. (1997) model that reproduces the so-called "Calzetti law". 
This configuration is supported by models of large-scale galactic winds driven by momentum deposition (Murray et al. 2005).

SMC-like dust seems to be present in Lyman-break galaxies at $z \sim 3$ as well (Vijh et al. 2003). This result is complemented by the finding of Noll \& Pierini (2005, NP05), based on spectroscopy instead of broad-band photometry. For a sample of 34 massive, UV-luminous galaxies at $2<z<2.5$, NP05 find that the majority of the objects with strongly reddened, restframe UV spectra have an extinction curve similar to the LMC curve. Conversely, the objects with the least reddened, rest-frame UV spectra seem to host SMC-like dust. This result strengthens the evidence for a difference in the properties of the dusty ISM at intermediate/high redshifts arising from previous, sometimes contradictory, results in the literature (e.g., Malhotra 1997; Pitman et al. 2000; Maiolino et al. 2001; Vernet et al. 2001; Hopkins et al. 2004; Savaglio \& Fall 2004; Wang et al. 2004; Wild \& Hewett 2005; York et al. 2006).

Here we extend the study of NP05 to a heterogeneous sample of massive, UV-luminous galaxies at $1<z<2.5$. This sample is three times larger than the NP05 one and probes the new redshift range $1<z<2$. Furthermore, the available ground-based spectroscopy allows us to date the oldest stellar populations at least in objects at $1<z<1.5$. We can also characterise the morphology of a galaxy across the whole redshift range in a reliable way from existing imaging with the ACS camera on board the Hubble Space Telescope (HST).

Throughout this paper $H_{0}=70 \mathrm{~km} \mathrm{~s}^{-1} \mathrm{Mpc}^{-1}, \Omega_{\Lambda}=0.7$, and $\Omega_{\mathrm{M}}=0.3$ are adopted. Photometry is given in the Vega magnitude system.

\section{The spectroscopic sample}

Inferring the presence of the broad dust absorption feature centred at $2175 \AA$ either directly (from individual and stacked spectra) or indirectly (with the method established in NP05, see Sect. 3.1) from galaxy spectra taken at optical telescopes limits the redshift range to $1<z<2.5$. The need for rest-frame UV spectra with a good continuum definition limits the sample to bright (and massive), star-forming galaxies. Finally, the need for a representative sample of such galaxies within this redshift range implies selection from different spectroscopic surveys.

For the total sample of 108 massive, star-forming galaxies at $1<z<2.5$ selected as described hereafter, Figs. 1 and 2 show the distribution of the $R$ magnitude (observed frame) as a function of redshift and the distribution in the $R-K_{\mathrm{S}}-K_{\mathrm{S}}$ colour-magnitude plane (observed frame), respectively. It is evident that our sample contains mostly objects with $23 \lesssim R \lesssim 24$ and $2<R-K_{\mathrm{s}}<5$ whatever the redshift, $R-K_{\mathrm{S}}$ turning bluer towards fainter $K_{\mathrm{s}}$ magnitudes. In general, the constraint on the $\mathrm{S} / \mathrm{N}$ of the optical spectra implies that galaxies at $1.5<z<2.5$ are more luminous in the rest-frame UV than those at $1<z<1.5$ (cf. Fig. 8), on average. In terms of star-formation activity and stellar mass, the total sample is less heterogeneous than what may be expected from the different selection criteria of the three surveys of origin (cf. Sect. 4.4). Table 1 lists basic properties of the sample galaxies. Hereafter we illustrate the different selection criteria and properties of the three subsamples.

\subsection{The FDF subsample}

In addition to the 34 galaxies at $2<z<2.5$ with $R<24.7$ investigated by NP05, we select 32 objects at $1<z<2$ with $R<24$ from the same $I$-limited FORS Deep Field (FDF) Spectroscopic

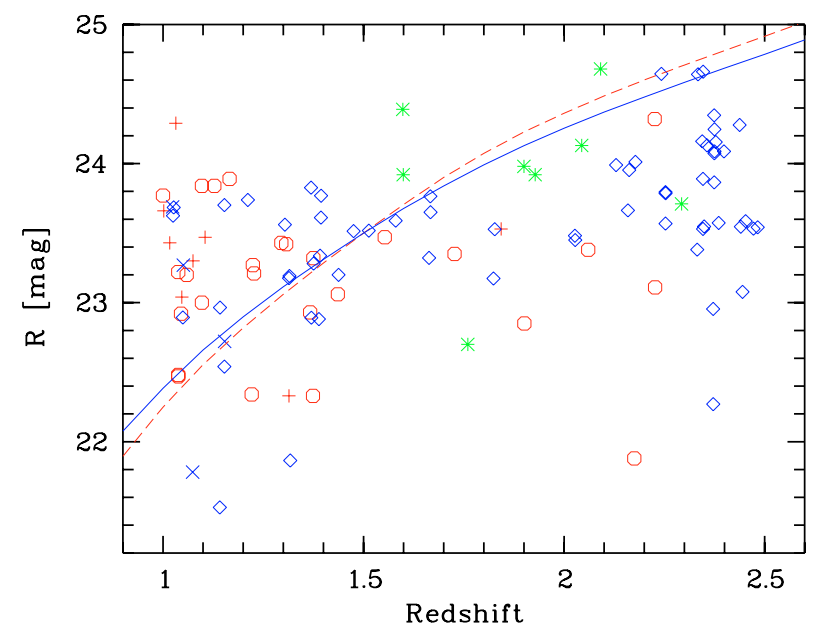

Fig. 1. $R$ magnitude versus redshift for the total sample of 108 actively star-forming galaxies at $1<z<2.5$ selected from the FDF Spectroscopic Survey (lozenges and crosses), the K20 Survey (circles and plus signs), and the GDDS (asterisks). Crosses, plus signs, and asterisks mark galaxies without a determination of the UV continuum slope. In order to illustrate selection biases, the curves show lines of constant luminosity for typical $1<z<1.5 \mathrm{FDF}$ (solid line) and K20 (dashed line) spectra. Both curves intersect at $R=23.5$ and $z=1.5$.

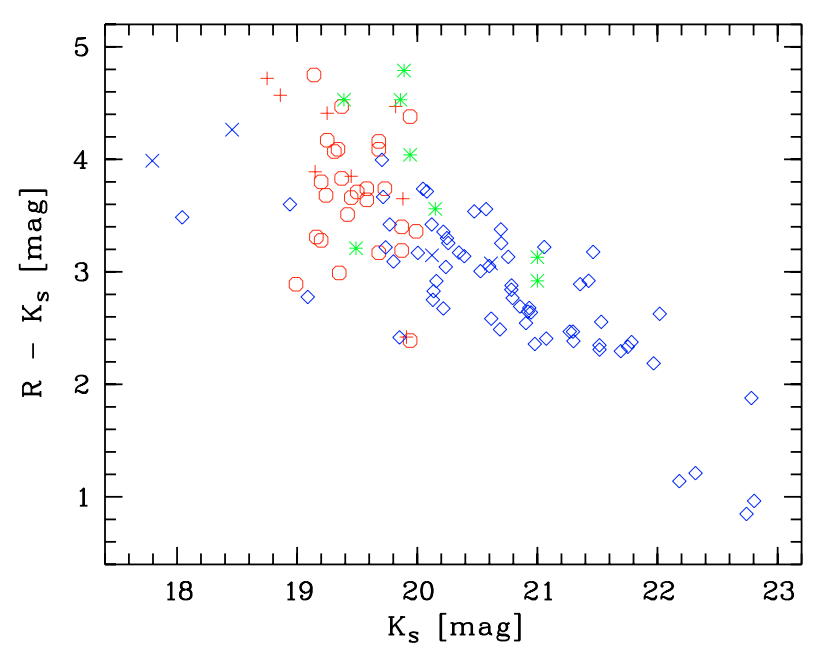

Fig. 2. $R-K_{\mathrm{s}}$ versus $K_{\mathrm{s}}$ magnitude for the total sample under investigation. Symbols are the same as in Fig. 1 .

Survey (Noll et al. 2004). Only galaxies with a dominant young stellar population (types III to V, see Noll et al. 2004) are considered. Basic aspects of the data reduction are illustrated in Noll et al. (2004) and NP05.

Ground-based optical/near-IR photometry in nine filters is available from Heidt et al. (2003) and Gabasch et al. (2004), respectively. The FDF was also imaged in the broad-band $F 814 W$ filter with the HST ACS camera. A $10 \sigma$ limit of 26 mag was reached with four WFC pointings of 40 min-exposure each. The data reduction was performed with the standard $C A L A C S^{1}$ pipeline, and the combined final mosaic was produced with the MultiDrizzle package (Mutchler et al. 2002).

\footnotetext{
1 www.stsci.edu/hst/acs/analysis
} 


\subsection{The K20 subsample}

We select 34 star-forming galaxies at $1<z<2.3$ from the spectroscopic catalogue of the K20 Survey in the Chandra Deep Field South (CDFS) and a field around the quasar 0055-2659 (Cimatti et al. 2002; Mignoli et al. 2005). Besides having $K_{\mathrm{s}}<20$, all the selected objects but two have $R<24$. This subsample comprises only K20 objects with a secure redshift and a suitable spectral coverage across $2175 \AA$ (basically from 1900 to $2450 \AA$ ). Galaxies with spectral energy distributions (SEDs) dominated by old stellar population (class 1 and 1.5, see Mignoli et al. 2005) are rejected.

In addition to the basic reduction described in Mignoli et al. (2005), the K20 spectra have been corrected for slit losses using the $R$ magnitude of the individual galaxies. Furthermore, they are corrected for Galactic extinction taking the MW extinction law of Cardelli et al. (1989) and the value of Galactic reddening $E(B-V)$ from Schlegel et al. (1998). Finally, they are mapped to the rest-frame, and smoothed to a similar resolution as for the FDF spectra (rest-frame 5 to $8 \AA$ ).

The CDFS was imaged with the HST ACS camera in four different filters as part of the ACS GOODS legacy programme (Giavalisco et al. 2004). We make use of the $F 775 \mathrm{~W}$ band images, where a $10 \sigma$ limit of $26.5 \mathrm{mag}$ was reached. For the data reduction, we refer the reader to Giavalisco et al. (2004).

\subsection{The GDDS subsample}

From the $I$ and $K_{\mathrm{s}}$-limited Gemini Deep Deep Survey (GDDS, Abraham et al. 2004), we consider eight additional star-forming galaxies at $1.5<z<2.3$ all of them having $R<24.7$ and $K_{\mathrm{s}} \lesssim$ 21.0. The limited redshift range is mainly due to the relatively narrow wavelength range (typically from 5500 to $9200 \AA$ ) of the red GMOS grating used in the GDDS. The GDDS subsample makes half of the total sample for $1.5<z<2.3$ and consists of galaxies with a prominent, young stellar population (class " 100 ", see Abraham et al. 2004).

The basic spectra of Abraham et al. (2004) have been reduced in the same way as described in Sect. 2.2 in order to meet the same standard as the spectra of the FDF and K20 subsamples (taken with VLT FORS).

\subsection{The reference sample of nearby starbursts}

We make use of the same reference sample of 24 local $(z \lesssim 0.02)$ starburst galaxies selected by NP05. For these starbursts, lowresolution IUE spectroscopy is available for the wavelength range between 1150 and $3350 \AA$.

Sample selection and data reduction are described in NP05.

\section{Data analysis}

The shape of the extinction curve at rest-frame UV wavelengths is constrained from a suitable parametric description of the UV SED of a galaxy. This method was introduced by NP05 and is briefly described in Sect. 3.1. Fundamental properties of a galaxy, like star-formation rate (SFR) and stellar mass, are estimated by model fitting the optical spectra (Sects. 3.2 and 3.3). Morphological information comes from model fits of the 2D surface brightness distribution of individual galaxies with diffraction-limited HST-ACS imaging (Sect. 3.4).

\subsection{A parametric description of the UV continuum}

NP05 introduced a parametrisation of the rest-frame UV SED of a galaxy based on five power-law fits to different sub-regions of the UV continuum of the same form as in Calzetti et al. (1994). Narrow wavelength regions affected by the presence of strong spectral lines are excluded from the fitting procedure for all parameters (cf. Calzetti et al. 1994; Leitherer et al. 2002b).

Two parameters are particularly suitable to constrain the slope of the extinction curve in the far-UV and the presence/absence of the broad absorption feature centred at $2175 \AA$. The first parameter characterises the apparent strength of the UV bump and is called $\gamma_{34}$. It is the difference between the continuum slopes measured at 1900-2175 $\left(\gamma_{3}\right)$ and 2175-2500 $\left(\gamma_{4}\right)$, respectively. A value of $\gamma_{34} \sim 1$ indicates the absence of the $2175 \AA$ feature. This is consistent with the presence of SMC-like dust in the ISM of a galaxy. Conversely, $\gamma_{34}<-2$ points to an extinction law which exhibits a significant UV bump. The second parameter gives the amount of reddening in the UV, according to the SHELL models of radiative transfer by Witt \& Gordon (2000). In fact, the relation between amount of dust or opacity and reddening is model dependent (e.g., Witt $\&$ Gordon 2000). These SHELL models with SMC dust are suitable to describe dust attenuation in local and high-redshift starbursts (Gordon et al. 1997; Vijh et al. 2003). Here the parameter estimating reddening in the UV is defined as the continuum slope measured at $1750-2600 \AA$, with the exclusion of the range $1950-2400 \AA$. This proxy for the UV reddening by dust is called $\beta_{\mathrm{b}}$ since it replaces the measure of UV-reddening $\beta$ adopted by NP05. In fact, $\beta$ is determined at $1250-1750 \AA$ and, thus, is not measurable in optical spectra of objects at $z<2$.

In order to establish $\beta_{\mathrm{b}}$ as a suitable alternative to $\beta$, we compare the values of these two parameters determined from synthetic SEDs (Fig. 3). We make use of different models combining stellar population evolutionary synthesis (Maraston 2005) and dust attenuation. For data-consistent ages of the stellar populations and metallicities (see Sect. 4.4), the variations in $\beta_{\mathrm{b}}$ are only of the order of 0.1 . Therefore, in Fig. 3 we assume the same properties for the stellar populations, i.e.: a continuous star-formation over $100 \mathrm{Myr}$ at a constant rate, a standard Salpeter (1955) initial mass function (IMF), and solar metallicity $Z_{\odot}$. Conversely, dust attenuation follows different prescriptions (see figure caption). In general, all models show that $\beta_{\mathrm{b}}$ and $\beta$ increase together when the opacity increases, with the only exception of models implementing the Witt \& Gordon (2000) SHELL configuration with a two-phase, clumpy medium and MW-type dust. In this case, $\beta_{\mathrm{b}}$ stays constant $(\sim-2.6)$ whereas $\beta$ increases when the opacity increases. This behaviour is due to the fact that in the spectral regions where $\beta_{\mathrm{b}}$ is defined, attenuation increases at the same rate with increasing opacity. The mapping of $\beta$ into $\beta_{\mathrm{b}}$ is model dependent, of course. An overall monotonic relation between $\beta_{\mathrm{b}}$ and $\beta$ holds even when more complex models assuming an age-dependent extinction are considered (see Sect. 4.3). The locus of these models in the $\beta-\beta_{\mathrm{b}}$ plane only moderately depends on the dust distribution, the dustclearing time scale, and the star-formation history. For instance, for composite Witt \& Gordon (2000) models with LMC-type dust and age-dependent extinction we typically find $\Delta \beta_{\mathrm{b}}$ to be less than 0.2 for constant $\beta$. In conclusion, we can take $\beta_{\mathrm{b}}$ as an alternative proxy for the UV-continuum reddening, though $\beta_{\mathrm{b}}$ has a lower dynamic range than $\beta$.

Furthermore, the choice of simple but not simplistic radiative transfer models to illustrate the relation between $\beta_{\mathrm{b}}$ and $\beta$ is 


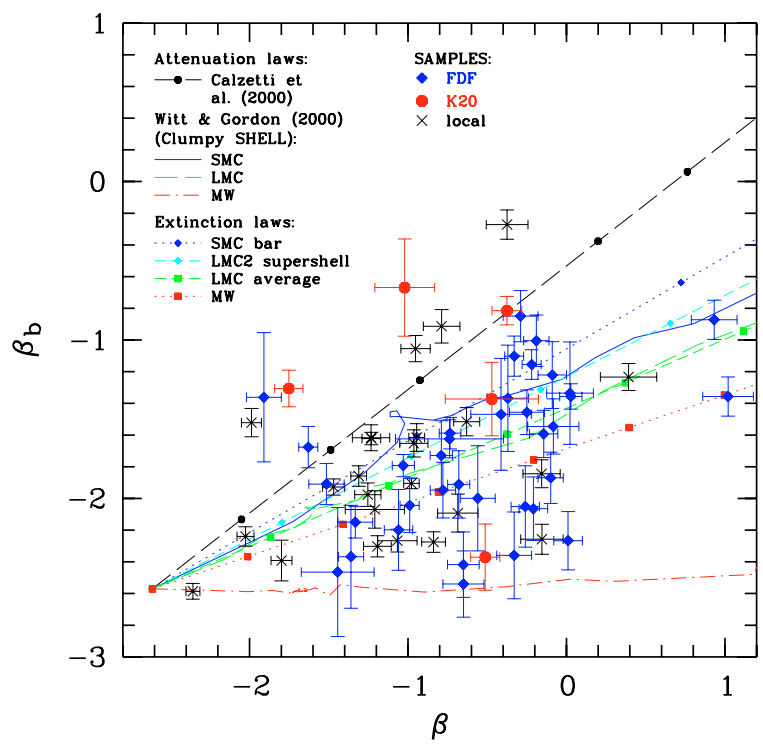

Fig. 3. Comparison of the two UV-continuum slope parameters $\beta$ $(1250<\lambda<1750)$ and $\beta_{\mathrm{b}}(1750<\lambda<2600)$ obtained from models combining stellar population evolutionary synthesis and dust effects as a function of the opacity of the dusty ISM. Dust attenuation is described either as for the empirical Calzetti et al. (2000) law (long dashed lines and circles) or as for the Witt \& Gordon (2000) radiative transfer models for the SHELL configuration of dust and stars and a two-phase, clumpy ISM with SMC- (solid lines), LMC- (long dashed lines), or MW-type dust (dash-dotted lines). Furthermore, we make use of a screen model plus the extinction laws of the SMC bar (dotted lines and lozenges), the LMC 2 supershell (dashed lines and lozenges), the LMC average (dashed lines and squares), and the MW average (dotted lines and squares) (Cardelli et al. 1989; Gordon et al. 2003). Symbols are plotted in intervals of $\Delta E(B-V)=0.1$. In all cases we adopt a Maraston (2005) model with a standard Salpeter IMF, constant SFR, age of $100 \mathrm{Myr}$, and solar metallicity. The diagram also shows parameter values measured for 24 local starburst galaxies (crosses), 33 FDF galaxies at $1.8<z<2.5$ (filled lozenges), and five K20 objects at $1.9<z<2.3$ (filled circles).

supported by the fact that most of the data points occupy the region delimited by these models in Fig. $3^{2}$. Simultaneous measurements of $\beta_{\mathrm{b}}$ and $\beta$ were possible for 38 galaxies at $1.8<z<$ 2.5 out of the FDF and K20 subsamples, and for the 24 local starburst galaxies. Part of the scatter of these data points reflects the dependence of $\beta_{\mathrm{b}}$ and $\beta$ from the dust properties of individual galaxies, as described by the models. Measurement errors in $\beta_{\mathrm{b}}$ contribute to this scatter as well. They are caused by the presence of residuals of night sky lines in the optical spectra obtained for the high-redshift galaxies, or by the uncertainties in the correction for Galactic extinction and the combination of the spectra of the IUE short- and long-wavelength channels for the nearby starbursts (see NP05).

\subsection{Star-formation rates}

For galaxies at $z>1.5$, the available optical spectra map restframe UV wavelengths only. These spectra are contrasted to a suite of synthetic SEDs across the mapped wavelength domain

${ }^{2}$ We note that the distribution of nearby starbursts in Fig. 3 shows several cases of significant displacement from the locus expected from the Calzetti law (Calzetti et al. 1994, 2000). This must not be surprising since the Calzetti law is a polynomial fit to the data, and, thus, expresses an average behaviour of dust attenuation in nearby starbursts across the whole wavelength range of applicability. excluding the range 1950-2400 $\AA$ and intrinsic (i.e., corrected for attenuation by internal dust) SFRs are determined as a bestfit solution. In particular, we build a grid of Maraston (2005) models with constant $\mathrm{SFR}^{3}$, an age between 10 and $1000 \mathrm{Myr}$, and metallicity equal to $0.5,1$, or 2 times solar $\left(Z_{\odot}\right)$. The ensuing SEDs are attenuated according to the Calzetti et al. (2000) law, where the optical colour excess $E(B-V)$ is the only free parameter. This choice is dictated by consistency with the literature and simplicity. Hence, the best-fit values of the intrinsic SFRs have to be taken "cum grano salis". In fact, on one hand, uncertainty is contributed by the unknown, true attenuation function that characterises individual objects, as discussed in Sect. 4. On the other hand, the data only roughly constrain age and metallicity of the models. Thus, the degeneracy between the analogous effects of age and dust reddening on the UV SED is not broken.

For galaxies at $z<1.5$, the available optical spectra do not map wavelengths $\lesssim 1500 \AA$. Therefore, we extrapolate the rest-frame UV SEDs of these galaxies down to the $\operatorname{Ly} \alpha$ line (1216 $\AA$ ) by fitting models in the UV domain excluding the range 1950-2400 $\AA$. To this purpose, it is sufficient to adopt a Maraston (2005) model with age of $100 \mathrm{Myr}$, solar metallicity, standard Salpeter IMF, and constant SFR. The ensuing SED is attenuated according to the Calzetti et al. (2000) law as before. The goodness of this procedure is evaluated by comparing the UV-continuum slope parameters for fitted synthetic rest-frame UV spectra and available observed spectra mapping the restframe UV down to Ly $\alpha$ (see Sect. 2). As a result, these fitted synthetic spectra exhibit bluer UV continua than the real spectra for $\lambda_{\min }>1500 \AA$. Hence, the original fitted values of $E(B-V)$ in the Calzetti law were corrected as a function of the measured value of $\beta_{b}(\langle\Delta E(B-V)\rangle=0.04)$ and new extrapolations were obtained. We estimate that the uncertainty in the narrow-band, rest-frame UV luminosity at $1500 \pm 20 \AA, L_{1500}$ (see Noll et al. 2004), associated with this extrapolation is about 0.04 dex.

As a consistency check, we estimate SFRs from the [O II] emission doublet at $3727 \AA$ (e.g., Kennicutt 1998; Kewley et al. 2004) for galaxies at $z<1.5$. We adopt the calibration of Kewley et al. (2004; see their Eq. (19)), assuming a nearly solar metallicity. We make the assumption that the values of the "instantaneous" SFR obtained from the [O II] line emission are statistically the same as the values of the SFR averaged over $100 \mathrm{Myr}$ obtained from the rest-frame UV continuum. Figure 4 shows that these two sets of estimated SFRs agree, if the stellar continuum-to-nebular line-emission reddening ratio is equal to $0.65(\sigma=0.08)$, the scatter in the SFR differences being of only 0.2 dex. This reddening ratio is close to those estimated by Calzetti et al. (2000) and Fernandes et al. (2005), i.e., $E(B-V)_{\text {stars }} / E(B-V)_{\text {gas }} \sim 0.44$ and 0.5 , respectively. It is significantly less than the value of 1 often assumed in the literature (e.g., Pierini et al. 2005; Erb et al. 2006b).

\subsection{Stellar masses}

Total stellar masses are taken from the literature. They were derived following status-of-art techniques that offer consistent results within the uncertainties of the photometric data and model assumptions. They are also consistent within the uncertainties of a few times 0.1 dex with the masses determined by our SFR estimates (see Sect. 3.2). For the FDF and K20 subsamples, stellar

\footnotetext{
3 Models with a SFR that declines exponentially as a function of time were also explored but they were poorly constrained by the data. Therefore they will not be discussed hereafter.
} 


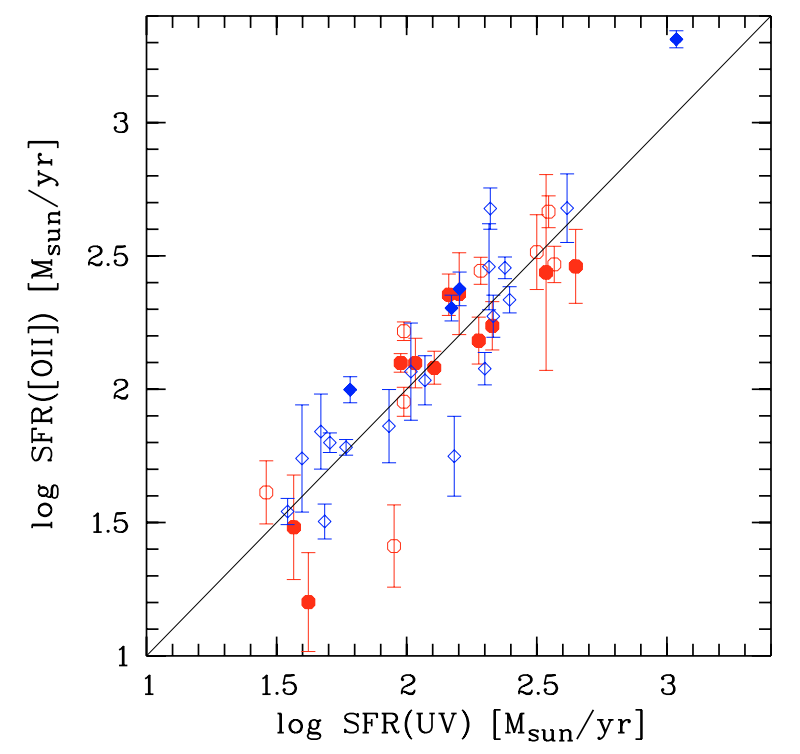

Fig. 4. Comparison of $S F R$ (UV) derived from model fits to the UV/optical spectra and $S F R$ ([O II]) derived from [O II] fluxes for the sample FDF (lozenges) and K20 galaxies (circles) at $1<z<1.5$. Objects with $\gamma_{34}<-2$ are marked by filled symbols. The $S F R$ ([O II]) were dust corrected using the Calzetti law as for the $S F R(\mathrm{UV})$. The best agreement between these values is obtained for a stars-to-gas reddening ratio $E(B-V)_{\text {stars }} / E(B-V)_{\text {gas }}=0.65$ and is reproduced here. Errors in the $S F R$ ([O II]) only express uncertainties in the [O II] flux.

masses come from Drory et al. (2005). These authors derived the most likely, total stellar masses and their uncertainties after fitting a large suite of two-component models to the FDF (Heidt et al. 2003) and CDFS multi-wavelength photometry (Salvato et al. in prep.). Each model consisted of a combination of two composite stellar populations: one given by a young (100 Myr) burst with constant SFR, the other by an older ( $\geq 500 \mathrm{Myr})$ burst with an exponentially declining SFR. Both components of the stellar continuum emission were attenuated independently according to Calzetti et al. (2000). For the GDDS subsample, total stellar masses come from Glazebrook et al. (2004). These authors derived the most likely, total stellar masses and their uncertainties by evaluating synthetic SEDs against the GDDS photometry of a galaxy to determine the mass-to- $K$-band luminosity ratio, and hence the mass. Two-component models plus a standard recipe for attenuation were adopted in a way analogous to Fontana et al. (2003) and Brinchmann \& Ellis (2000).

\subsection{Morphological parameters}

The package GIM2D (Simard et al. 1999) is used to fit pointspread function (PSF) convolved Sérsic (1968) profiles to the two-dimensional surface brightness of each object, down to a limit of $F 814 W=24$ (FDF) and $F 775 W=24.5$ (CDFS). The Sérsic model contains seven free parameters: total intensity, scale-length of the semi-major axis, ellipticity $e$, position angle, index $n_{\text {ser }}$, and model $x, y$ centring. The most valuable parameter is $n_{\mathrm{ser}}$, which determines the shape of the profile. For each fit, GIM2D automatically determines the initial values and limits for the parameter space to be explored using a segmentation mask for deblending multiple objects. This mask is provided by the standard pipeline for the automated source extraction and photometry SExtractor (Bertin \& Arnouts 1996). The PSFs used to convolve the model profiles were obtained for each tile by stacking about 10 high $\mathrm{S} / \mathrm{N}$ isolated stars. We put extensive efforts in understanding and quantifying how various kinds of systematics (e.g., PSF variations over the field of view or dependences on the $\mathrm{S} / \mathrm{N}$ ratio) could affect the modelling results. To this purpose, simulated images with the same characteristics of the true ones were produced and analysed in the same way as for the ACS images. GIM2D also computes the two indices $R_{\mathrm{T}}$ and $R_{\mathrm{A}}$ from the thumbnail residual image. These indices provide an estimate of the overall smoothness of a galaxy image with respect to the fitting model. In other words, they estimate the residual substructure like spiral arms in nearby late-type galaxies (see the seminal study of Elmegreen et al. 1992), peculiarity/asymmetry in the distribution of giant star-forming regions, or interaction/mergers in high-redshift galaxies (e.g., Schade et al. 1995).

Furthermore, we characterise the (potentially complex) morphologies of our galaxies by means of the model-independent parameters concentration $C$ and asymmetry $A$. The $C$ - $A$ method was developed in the mid-nineties by Abraham et al. (1994, 1996). Subsequent works (e.g., Wu 1999; Conselice et al. 2000, 2003; Menanteau et al. 2006) have shown that a better morphological classification is obtained by choosing an image pivot point which minimises the measured asymmetry. We make use of the CAS parametrisation as proposed and described in detail by Conselice et al. $(2000,2003)$. Here it is important to say that early-type galaxies have larger concentration and lower asymmetry indices than later ones.

\section{Results}

Hereafter we show that dust with a UV bump can be present in massive, star-forming galaxies at $1<z<2.5$ (Sect. 4.1). Sect. 4.2 contains reasons why sample selection criteria can hinder the inference of the presence of the dust components responsible for the absorption excess at $2175 \AA$ (i.e., the carriers of the UV bump) from a similar spectral analysis of high-redshift galaxies. We interpret our results in Sect. 4.3. Then we investigate how the presence of different extinction curves in highredshift galaxies depends on global galaxy properties such as SFR and total stellar mass (Sect. 4.4). Finally, we discuss the effects of topology and metal enrichment of the ISM on the observed strength of the UV bump (Sect. 4.5).

\subsection{Dust with a pronounced UV bump already exists at high redshift}

Figure 5 shows the proxy for the strength of the $2175 \AA$ feature, $\gamma_{34}$, versus the proxy for reddening in the UV, $\beta_{\mathrm{b}}$, for three different redshift bins, i.e.: $1<z<1.5$ (40 objects), $1.5<z<2$ (10 objects), and $2<z<2.5$ (38 objects). Tracks for the models described in Sect. 3.1 are also plotted. The comparison between the distribution of the observed quantities and the expected ones clearly shows that dust with a UV bump and, thus, different extinction curves (see Sect. 4.3) do exist at high redshift. Now this holds also for $1<z<1.5$ (cf. NP05).

Galaxies at $1<z<1.5$ exhibit the widest range in the observed strength of the UV bump $\left(-8<\gamma_{34}<1\right)$. According to the models described in Sect. 3.1, dust with a significant UV bump (i.e., $\gamma_{34}<-2$ ) characterises $58 \%$ of the $19 K_{\mathrm{s}}$-selected K20 galaxies and $19 \%$ of the $21 \mathrm{I}$-selected FDF galaxies at these redshifts ${ }^{4}$. This drop is partly caused by selection effects (see Sect. 4.2). In fact about $29 \%$ of the galaxies in the original FDF photometric sample with $1<z_{\text {phot }}<1.5$

\footnotetext{
4 We note that a MW-like extinction law is directly suggested only for CDFS-00525.
} 

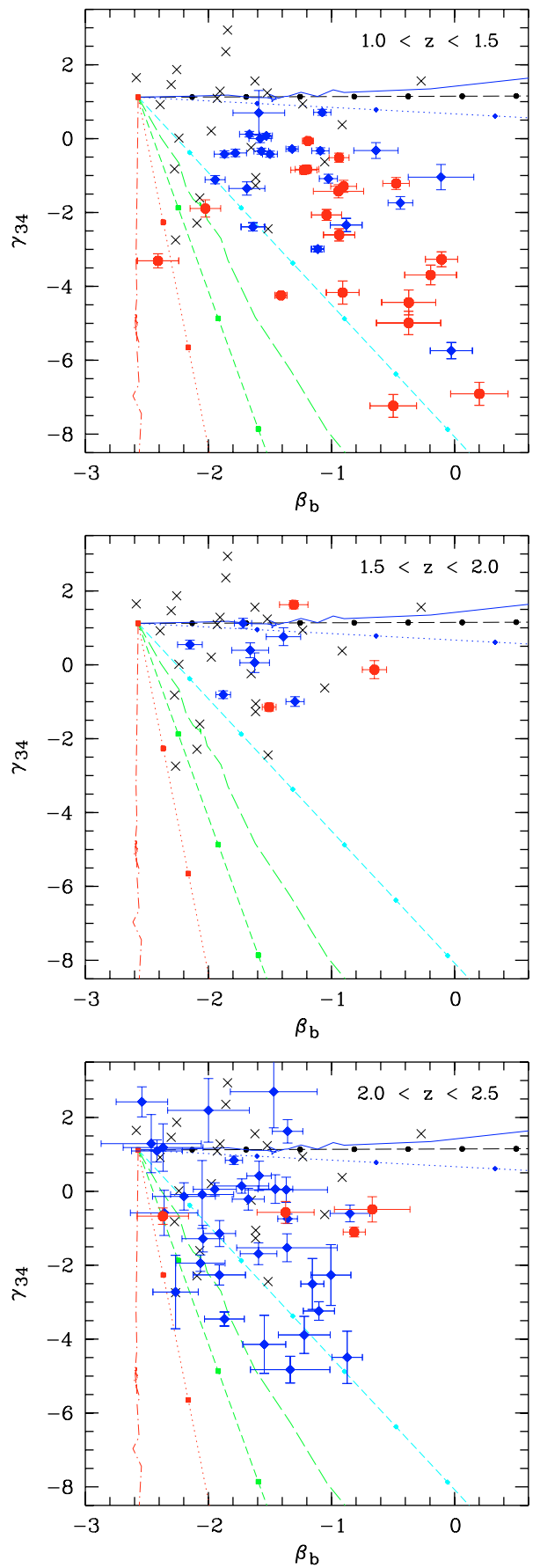

Fig. 5. Plot of the proxies for the $2175 \AA$ feature $\left(\gamma_{34}\right)$ and reddening in the UV $\left(\beta_{\mathrm{b}}\right)$ for the FDF (lozenges) and K20 (circles) galaxies listed in Table 1 for which both parameters were determined. The panels show the distribution of objects at $1<z<1.5$ (top), $1.5<z<2$ (middle), and $2<z<2.5$ (bottom), together with that of the 24 local starburst galaxies (crosses) as a reference. All panels reproduce model tracks obtained for different descriptions of dust attenuation as a function of $E(B-V)$ (see Fig. 3). We recall that more negative values of $\gamma_{34}$ indicate stronger $2175 \AA$ absorption features.

and $R<24$ have $K_{\mathrm{s}}<20$ (Heidt et al. 2003; Gabasch et al. 2004). The fraction of FDF galaxies with $\gamma_{34}<-2$ reaches $33 \%$, if only the six objects with $K_{\mathrm{s}}<20$ are considered. This suggests that dust with a significant UV bump can be present in at least half of the $K_{\mathrm{s}}$-bright, star-forming galaxies at $1<z<1.5$, whatever the selection criterion.

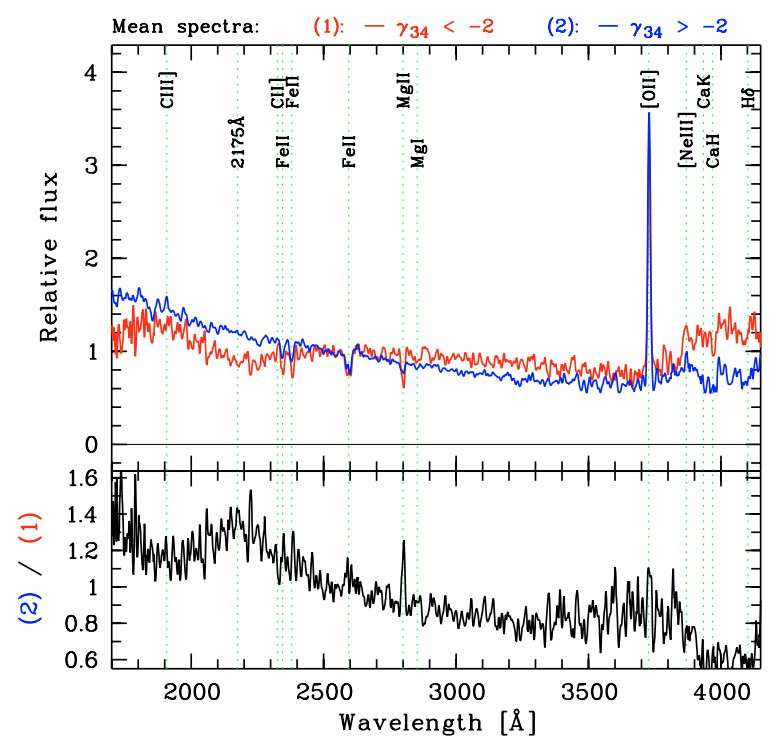

Fig. 6. Comparison of $K_{\mathrm{s}}$-weighted composite spectra of $1<z<1.5$ galaxies with $R<24$ exhibiting $\gamma_{34}>-2$ (blue or dark grey) and $\gamma_{34}<-2$ (red or grey), respectively (top). The ratio of both composites, normalised at $2400-2570 \AA$, is also shown (bottom).

Furthermore, Fig. 5 shows that the $2175 \AA$ absorption feature tends to be associated with larger amounts of reddening at UV wavelengths. This confirms the result of NP05. Interestingly the largest amounts of reddening appear in the lowest redshift bin. When we combine the K20 and FDF objects with $1<z<1.5$ to determine the $K_{\mathrm{s}}$-weighted ${ }^{5}$ mean value of $\gamma_{34}$, we find that $\left\langle\gamma_{34}\right\rangle=-0.44 \pm 0.18$ if $\beta_{\mathrm{b}}<-1.5$, whereas $\left\langle\gamma_{34}\right\rangle=-3.48 \pm 0.51$ if $\beta_{\mathrm{b}}>-0.5$. Consistently, in the same redshift bin, about $80 \%$ of the $K_{\mathrm{s}}$-bright galaxies with $\beta_{\mathrm{b}}>-0.5$ exhibit evidence of dust with a UV bump, but only about $40 \%$ of those with $\beta_{\mathrm{b}}<-0.5$ do so.

The evidence of a UV bump $\left(\gamma_{34}<-2\right)$ in Fig. 5 is made clearer by the comparison between the $K_{\mathrm{s}}$-weighted, composite spectra of galaxies at $1<z<1.5$ with $R<24$ and $\gamma_{34}>-2$ or $\gamma_{34}<-2$ (Fig. 6). An analogous proof was given by NP05 for their sample of FDF UV-luminous galaxies at $2<z<2.5$ (also reproduced in Fig. 5). Owing to the different mapping of the restframe UV domain, the composite spectra in Fig. 6 show remarkable differences in the strength of the Balmer/4000 $\AA$ break and in the $\mathrm{Mg}$ II doublet at $2800 \AA$. The origin of these differences is discussed in Sect. 4.4.

\subsection{Selection effects on the detection of the UV bump}

In Fig. 5, the few galaxies at $1.5<z<2$ do not seem to possess significant $2175 \AA$ features in their spectra, which is at odds with the UV-bump detections at lower and higher redshifts. Hence, these objects may not represent the full population of massive, star-forming galaxies at $1.5<z<2$, though their statistics is poorer than for objects in the other two redshift bins. Figure 8 shows that the sample galaxies at $1.5<z<2.2$ (all exhibiting $\gamma_{34}>-2$ ) have rest-frame luminosities at $1500 \AA$ that are on average three times higher than those of the galaxies with $\gamma_{34}<-2$ at $1.0<z<1.5$ but slightly less luminous than those with an

${ }^{5}$ For subsamples the weight of $K_{\mathrm{s}}$-bright objects differs from the value of $29 \%$ for the whole sample owing to the different distributions of the $K_{\mathrm{s}}$-bright and $K_{\mathrm{s}}$-faint galaxies as a function of individual selection quantities. 


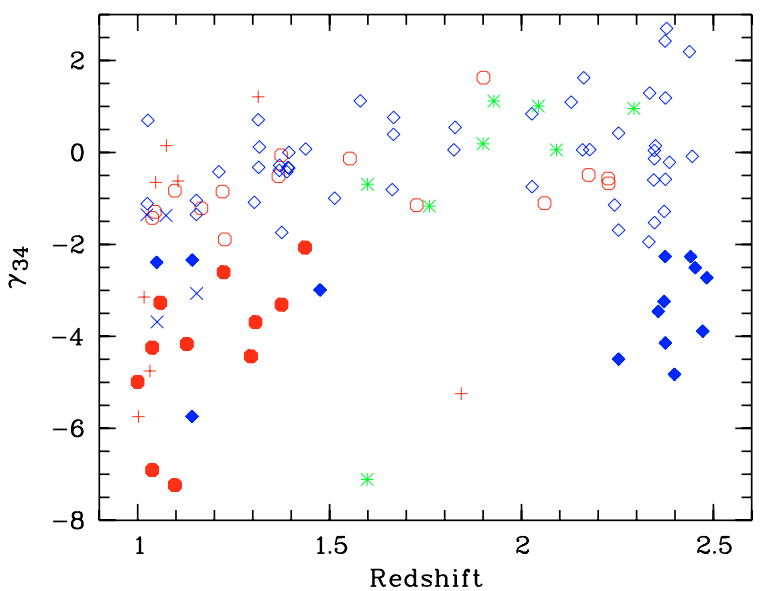

Fig. 7. The distribution of $\gamma_{34}$ values as a function of redshift for the full sample of 108 massive, star-forming galaxies at $1<z<2.5$ selected from the FDF Spectroscopic Survey (lozenges and $\times$ ), the K20 Survey (circles and + ), and the GDDS (asterisks). Crosses and asterisks mark galaxies with no possible determination of the UV continuum slope $\beta_{\mathrm{b}}$ owing to a limited spectral coverage. Filled symbols indicate objects with $\gamma_{34}<-2$, i.e., with an extinction curve exhibiting a significant UV bump.

evident UV bump at $2.3<z<2.5$ (34.02 \pm 0.06 versus $34.16 \pm 0.04$ in $\log L_{1500}-$ in [W/Å], respectively). This suggests that our selection can miss galaxies with a low, intrinsic UV luminosity and a significant UV bump, such as those typically found at $1.0<z<1.5$. However, it could also miss objects with a high, intrinsic UV luminosity and $\gamma_{34}<-2$, such as those found at $2.0<z<2.5$. This can be understood if the presence of a significant UV bump is indeed associated with a larger amount of attenuation at UV wavelengths, as suggested by the model interpretation of Fig. 5. In fact, the sample galaxies at $1.5<z<2.2$ are mostly selected in $I$ band and exhibit $R<24$ (cf. Sect. 2). Now, we note that the $R$ broad-band filter maps exactly the spectral region across the UV bump for $1.5<z<2.2$. As a check of this hypothesis, we plot the distribution of $\gamma_{34}$ as a function of redshift for the full sample of 108 massive, star-forming galaxies at $1<z<2.5$. With respect to Fig. 5, Fig. 7 contains additional 20 galaxies with no measurement of $\beta_{\mathrm{b}}$. Among the 26 galaxies at $1.5<z<2.2$, there are only two with $\gamma_{34}<-2$ against an expected number of $8 \pm 3$ (corresponding to a fraction of about $30 \%$ as from Sect. 4.1). For one (CDFS-00295) the determination of its spectroscopic redshift is uncertain. The other (SA15-4762) has $R=24.4$, i.e., it is just fainter than the limiting magnitude $(R=24)$ of the FDF and K20 subsamples. We conclude that selection effects can remove from the resulting sample very dusty galaxies with dust similar to the LMC or MW at $1.5<z<2.2$.

\subsection{Extinction curve and dust/stars configuration}

In the previous analysis as well as in NP05, it was assumed that dust attenuation in massive, UV-luminous galaxies at high redshifts is described by the Witt \& Gordon (2000) Monte Carlo calculations of radiative transfer for the SHELL configuration. In particular, a two-phase, clumpy, dusty medium is assumed to surround all stars, whatever the ages of the stellar populations. If this assumption holds, the interpretation of our results is straightforward: different mixtures of dust produce extinction curves that are intermediate between the SMC and LMC average extinction curves.

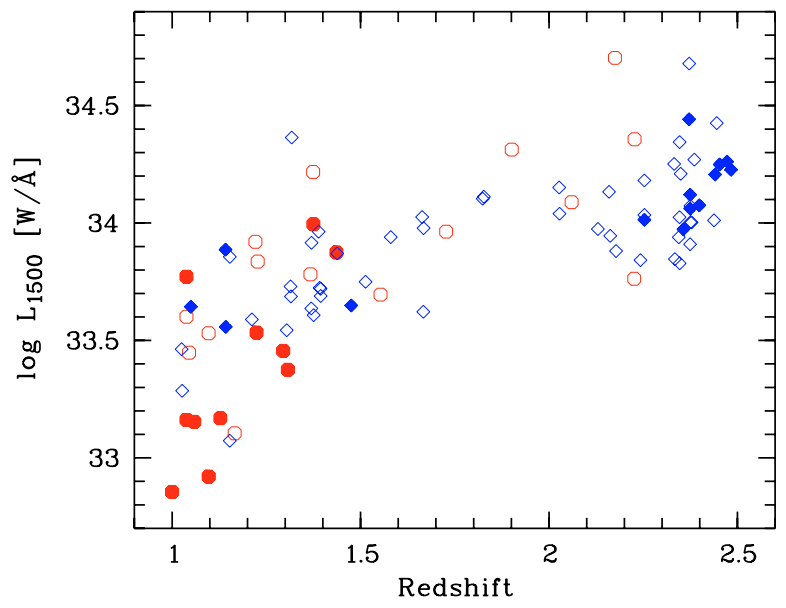

Fig. 8. Luminosity at $1500 \AA$ versus redshift for 88 galaxies of the FDF Spectroscopic Survey (lozenges) and the K20 survey (circles). Galaxies with $\gamma_{34}<-2$ are marked by filled symbols.

However, the previous assumption on the dust/stars configuration can sound too simple and, thus, be challenged. Therefore, hereafter we discuss two dust/stars configurations that differ substantially from each other and from the SHELL configuration in Witt \& Gordon (2000). In the first case, we consider a disc geometry together with a different distribution of stars with respect to the one of the dust according to the different ages of the stellar populations. This effect is known as the "age-dependent extinction" (Silva et al. 1998; Tuffs et al. 2004; Pierini et al. 2004; Panuzzo et al. 2007). In the second case, we assume that any of our sample galaxies can be ideally pictured as a system where stellar populations younger than a certain age (i.e., the dust clearing time $t_{\mathrm{c}}$ ) are enshrouded by dust, while older ones live in a dust-free environment.

We consider two different sets of models describing dust attenuation for a disc geometry, namely the "Sc model" from the GRASIL library (Silva et al. 1998) and the disc models with a two-phase, clumpy, dusty ISM in Pierini et al. (2004). In general, the GRASIL radiative transfer models describe the fact that new stars are born inside molecular clouds and at later times either get rid of their parent environment or move out of it. The radiative transfer models described in Pierini et al. (2004) account for the fact that younger stellar populations are more attenuated by dust than older ones by setting the scale-height of the distribution of photons equal to lower values the shorter the wavelength. In both cases a MW-like extinction curve is assumed. Three values of the inclination of the disc are considered in each set of models, namely 0 (i.e., a face-on view), 75 (or 70), and 90 (i.e., an edge-on view) degrees. Furthermore, six different values of the opacity are considered in the second set of models. In both cases, intrinsic SEDs are computed for a constant SFR, solar metallicity, and ages of 0.6 or 2 Gyr. As Fig. 9 shows, the models do not span a domain as wide as that of the data in the $\beta_{\mathrm{b}}-\gamma_{34}$ plane (cf. Fig. 5). In particular, they are confined to $-2.5 \leq \gamma_{34}<1$ although they assume an extinction curve similar to the average MW one. Differences do exist however. The GRASIL Sc models span a limited range in $\beta_{\mathrm{b}}$ as a function of the inclination of the disc with respect to the disc models described in Pierini et al. (2004), once the opacity is fixed. Assuming the presence of dust with a UV bump and a smaller scattering albedo for a shorter wavelength (except for the UV-bump range) would broaden the range in $\beta_{\mathrm{b}}$ spanned by the previous models but would not move any of them to the region of the highest values of $\beta_{\mathrm{b}}$ and the 


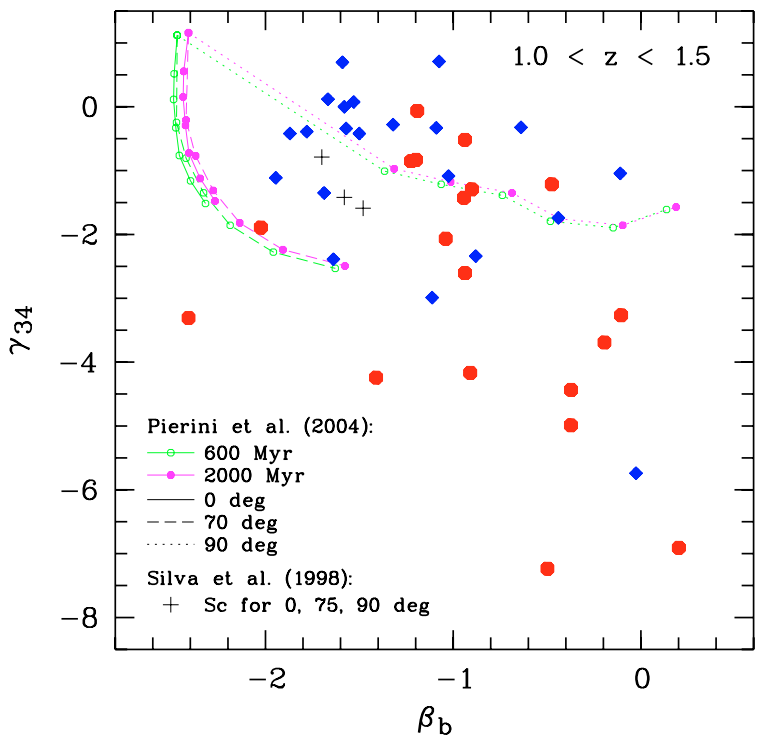

Fig. 9. Comparison among models where stars and dust are distributed in a disc. First, dust attenuation is described as in Pierini et al. (2004), assuming inclinations of 0 (solid line), 70 (dashed line), and 90 deg (dotted line) and continuous star-formation phases of 600 (small open circles) or $2000 \mathrm{Myr}$ (small filled circles). The $V$-band opacities plotted are $0,0.5,1,2,4,8$, and 16 . Second, we show Sc-galaxy GRASIL models (Silva et al. 1998) for inclinations of 0,75, and $90 \mathrm{deg}$ (crosses, from the left to the right, respectively).

lowest values of $\gamma_{34}$ (cf. Fig. 11 in Inoue et al. 2006). In conclusion, the comparison of observed values and predictions from a more physical dust/stars configuration for a disc geometry leads us to the conclusion that especially those galaxies with $\gamma_{34}<-2$ seem to have dust with a significant UV bump that is partly distributed out of the disc mid-plane or at least embeds the stellar populations responsible for the UV emission. This holds whatever the behaviour of the dust scattering albedo.

Alternatively, we assume that in our high-redshift galaxies new stars are born in "macro-regions" where the dusty ISM is cleared out after a time $t_{\mathrm{c}}$, which ends the star-formation activity there. For illustrative purposes only, we make the simplistic case that the stellar populations younger than the dust clearing time are screened by dust while the older ones do not suffer from extinction at all. As for the value of $t_{\mathrm{c}}$, we do not take the time scale of the dispersal of the parent molecular cloud by an individual supernova, as in Silva et al. (1998) or in Tuffs et al. (2004), which is of the order of a few Myr (e.g., Leitherer et al. 2002a). Conversely, we picture dust clearing as a collective phenomenon and set $t_{\mathrm{c}}$ equal to 100 or $400 \mathrm{Myr}$. Dust attenuation is described as for the SHELL configuration in Witt \& Gordon (2000), assuming either a homogeneous or two-phase, clumpy ISM with LMC-type dust. Only values of opacity giving a maximum attenuation at $1500 \AA$ of $5 \mathrm{mag}$ are considered. Furthermore, we consider Maraston (2005) stellar population synthesis models with maximum ages of $1 \mathrm{Gyr}$ and fixed, solar metallicity (see Sect. 4.4.1). The total stellar mass produced by the extinguished stellar population $M_{\text {ext }}$ is fixed to be half or twice the mass produced by the unextinguished population $M_{\text {unext }}$. The SFR is, therefore, changed (divided by a factor from 0.75 to 18 ) after a time equal to $t_{\mathrm{c}}$. Figure 10 shows a comparison of the previous models and the data in the $\beta_{\mathrm{b}}-\gamma_{34}$ plane, only for galaxies at $1<z<1.5$. Remarkably, this set of models spans the region defined by the observed measurements. For low opacities the light from extinguished, young stars dominates the UV spectra and

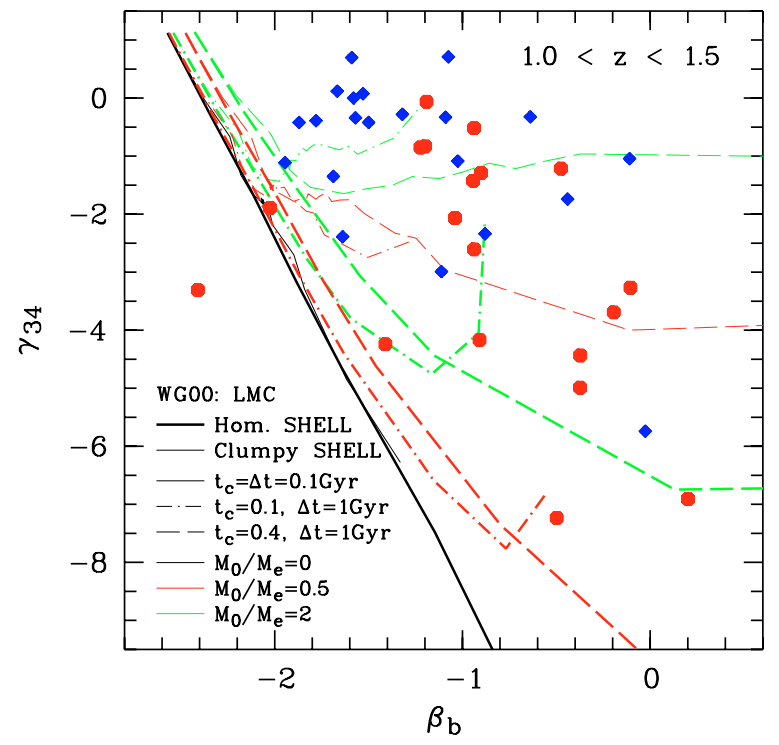

Fig. 10. Comparison between the distribution in the $\beta_{\mathrm{b}}-\gamma_{34}$ plane of observed galaxies at $1<z<1.5$ and models where the star-formation activity takes place in "macro-regions" until dust is cleared out there. Dust attenuation is described as in the Witt \& Gordon (2000) SHELL configuration, where the ISM is either two-phase, clumpy (thin lines) or homogeneous (thick lines) and contains average LMC-type dust. As for $t_{\mathrm{c}}$ (see Sect. 4.3), it is set equal to either $100 \mathrm{Myr}$ (dash-dotted and solid lines) or $400 \mathrm{Myr}$ (dashed lines). Star-formation goes on for a time equal to the dust clearing time $t_{\mathrm{c}}$ (solid, black lines; cf. Fig. 5) or to 1 Gyr. For the latter the total stellar mass produced by the extinguished stellar population $M_{\text {ext }}$ is fixed to be half (green or light grey lines) or twice (red or dark grey lines) the mass produced by the unextinguished population $M_{\text {unext }}$. These constraints lead to $S F R_{\text {ext }} / S F R_{\text {unext }}$ between 0.75 and 18. Three of four star-formation scenarios prescribe the instantaneous SFR at $t(z)$ to be above the SFR averaged over the age of the system.

the observed strength of the UV bump increases with UV reddening. For higher opacities the contribution of the unobscured, older stars becomes more and more important till $\gamma_{34}$ stalls or the strength of the $2175 \AA$ feature even decreases ${ }^{6}$. The latter trend is more striking for galaxies with short dust-clearing time scales. The most negative $\gamma_{34}$ in our data can only be modelled assuming little impact of nearly-unobscured intermediate age stars on the UV SEDs and the presence of an almost perfect dust screen for the obscured stellar populations. LMC-type dust or dust with even stronger UV bumps are required.

In conclusion, age-dependent extinction scenarios increase the probability of the presence of dust with a significant UV bump in our sample galaxies.

\subsection{Extinction curve and global galaxy properties}

The carriers of the UV bump exist in galaxies belonging to different subsamples. $2175 \AA$ features traced by $\gamma_{34}<-2$ are found for about $38 \%$ of the FDF galaxies at $2.3<z<2.5$. They exist as well in the spectra of about $25 \%$ of the $K_{\mathrm{s}}$-weighted combined subsample of FDF and K20 galaxies with $R<24$ at $1<z<1.5$. This fraction jumps to about $52 \%$ if one considers only $K_{\mathrm{s}}$-bright galaxies at these redshifts (Sect. 4.1). On the other hand, Fig. 8 shows that galaxies at $1<z<1.5$ exhibit lower luminosities at $1500 \AA$ than those at $2<z<2.5$, in general. For objects

\footnotetext{
6 An analogous decrease of the observed strength of the UV bump (when the fraction of less obscured stars older than $t_{\mathrm{c}}$ but still emitting UV photons increases) was already found by Panuzzo et al. (2007).
} 
with evidence of dust with a UV bump, $L_{1500}$ drops by a factor of 4-5. This suggests that the reasons for the presence and/or survival of the carriers of the UV bump in high-redshift galaxies are different, at least in part, at different redshifts.

Hereafter we investigate if, more generally, the extinction curve depends on global characteristics of a high-redshift galaxy like properties of the stellar populations, SFR, stellar mass, and morphology. We will consider six subsamples, which are classified by redshift, UV-continuum slope, and observed strength of the UV bump. In particular, we will compare a sample at $1<z<1.5$ ( $K_{\mathrm{s}}$-weighted, see Sect. 4.1) and one at $2<z<2.5$ (only FDF galaxies). Both samples are divided into three subsamples each. First, we define "blue" galaxies, i.e., galaxies with blue UV continua. For $2<z<2.5$, we take the same classification as introduced by NP05, i.e., the "blue" galaxies (16 objects) have $\beta<-0.4$. For $1<z<1.5$ we take $\beta_{\mathrm{b}}<-1.5$ (13 objects). The remaining, so-called "red" galaxies are divided into subsamples for $\gamma_{34}>-2(14$ objects at $2<z<2.5$ and 9 ones at $1<z<1.5)$ and $\gamma_{34}<-2$ (13 and 9 objects, respectively), since galaxies with relatively strong $2175 \AA$ features are mostly "red", i.e., $\beta>-0.4$ and $\beta_{\mathrm{b}}>-1.5$, respectively. We choose $\beta_{\mathrm{b}}=-1.5$ for $1<z<1.5$ since it is consistent with the discriminating value of $\beta$ for the higher-redshift galaxies. In fact, for those objects at $2<z<2.5$ with a simultaneous measurement of $\beta$ and $\beta_{\mathrm{b}}$ we find $\beta-\beta_{\mathrm{b}} \sim 1$. Comfortably, an analogous difference is obtained for the reference sample of nearby starbursts. By chance, $\beta_{\mathrm{b}}=-1.5$ divides the FDF subsample of galaxies at $1<z<1.5$ into blue and red subsamples of similar sizes (11 and 10 objects, respectively).

\subsubsection{Stronger $2175 \AA$ features in more "mature" galaxies}

Fitting the available spectra with the models described in Sect. 3.2 gives ages of less than $100 \mathrm{Myr}$ (for a constant SFR) for the majority of the sample galaxies. This simply confirms the star-forming nature of the sample galaxies. Ages of more than $1 \mathrm{Gyr}$ were determined for high-redshift star-forming galaxies like ours by Erb et al. (2006b) and Glazebrook et al. (2004), based on broad-band SEDs mapping the full rest-frame optical domain.

For galaxies at $z<1.3$, the spectra map also rest-frame optical wavelengths, reaching the region of the Balmer/4000 break, where the index D4000 is defined (Hamilton 1985; Balogh et al. 1999; Kauffmann et al. 2003). This spectral index is particularly useful to constrain ages $\gtrsim 1 \mathrm{Gyr}$, also at high redshifts (e.g., Le Borgne et al. 2006). We adopt the definition of D4000 by Balogh et al. (1999), where two fluxes (per unit of frequency) are measured in the wavelength windows 3850-3950 and 4000-4100 $\mathrm{A}$, bracketing the Balmer/4000 $\AA$ break. We measure a typical flux ratio of about 1.1 , which translates into an average age of $50_{-35}^{+150} \mathrm{Myr}$ for Maraston (2005) models with constant SFR and attenuated according to the Calzetti law, which provides lower age limits (see caption of Fig. 11). In the complementary dust-free case, this estimate increases to $250_{-170}^{+550} \mathrm{Myr}$. For more complex star-formation histories, including both a monotonic behaviour of the SFR as a function of time and a random burst of star-formation (cf. Kauffmann et al. 2003), a value of $\mathrm{D} 4000 \sim 1.1$ corresponds to a median age of the models equal to $630 \mathrm{Myr}$ (A. Gallazzi, priv. comm.). The ages of the latter models have a large scatter though, ranging from $126 \mathrm{Myr}$ to 4 Gyr. True characteristic ages will lie somewhere in between the dusty and dust-free cases.

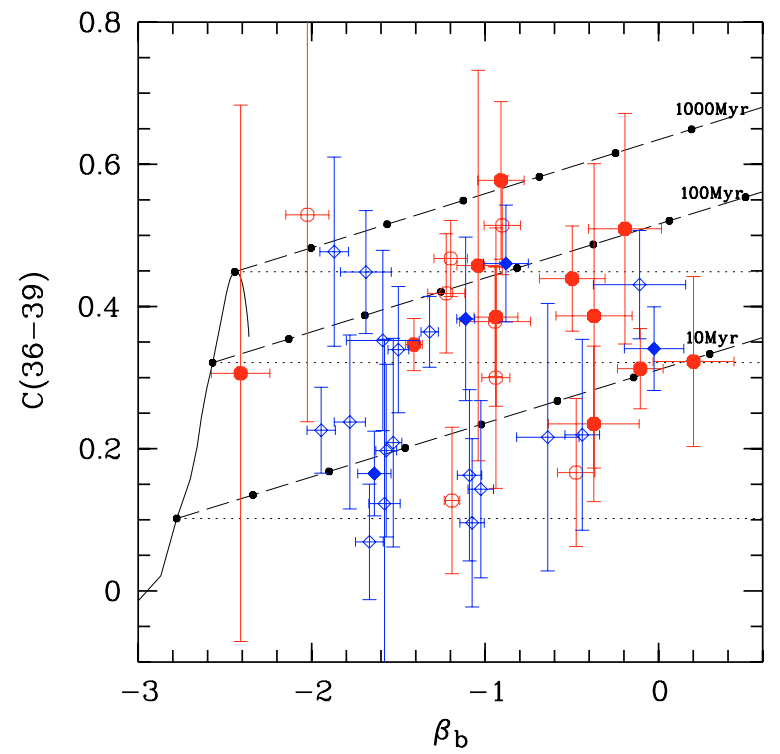

Fig. 11. Balmer-break proxy $C(36-39)$ versus reddening measure $\beta_{\mathrm{b}}$ for the sample FDF (lozenges) and K20 galaxies (circles) with $1<z<1.5$. Objects with $\gamma_{34}<-2$ are marked by filled symbols. Different Maraston (2005) models for continuous star-formation and solar metallicity are shown for estimating the age of the stellar population. The dashed lines indicate the effect of continuum reddening by a Calzetti et al. (2000) law on $\beta_{\mathrm{b}}$ and $C(36-39)$ for ages of $10,100,1000 \mathrm{Myr}$. The small filled circles are plotted in intervals of $\Delta E(B-V)=0.1$. Attenuation via the Calzetti law produces a stronger dependence of $C(36-39)$ on $\beta_{\mathrm{b}}$ than most other attenuation laws used in this study (see Fig. 3). Therefore, the estimated ages would typically increase by a factor of about 2 using SMC and LMC extinction curves. The dust-free cases for the three considered ages are indicated by the dotted lines.

Characteristic ages can be determined for all objects at $z<$ 1.5 , if one considers the Balmer-break sensitive pseudo-colour $C(36-39)$, defined as the flux ratio (in magnitudes) between the fluxes (per unit of frequency) at 3500-3700 $\AA$ and 3750-3950 (cf. Mignoli et al. 2005). In this case, we derive a mean age of $25_{-5}^{+10} \mathrm{Myr}$ from the comparison between data and models (Fig. 11). If only galaxies at $z<1.3$ are considered, the mean age increases to $80_{-30}^{+50} \mathrm{Myr}$. This is consistent with the indications from D4000. As also suggested by Fig. 6, the mean ages of galaxies with $\gamma_{34}<-2$ are greater than those of galaxies with $\gamma_{34}>-2\left(50 \pm 15 \mathrm{Myr}\right.$ and $20_{-5}^{+10} \mathrm{Myr}$, respectively). This trend is strengthened in the dust-free case, where upper limits to the characteristic age of the stellar populations are equal to $380_{-160}^{+270} \mathrm{Myr}$ and $80_{-30}^{+50} \mathrm{Myr}$ for galaxies with $\gamma_{34}<-2$ and $\gamma_{34}>-2$, respectively. In conclusion, the analysis based on spectroscopic indices reveals the presence of intermediate-age (0.2 to $1-2$ Gyr-old) stellar populations in galaxies at $1<z<1.5$. Furthermore, it suggests that galaxies with evidence of a UV bump at these redshifts have a larger fraction of intermediate-age stars and/or are older than those lacking an evident UV bump.

Standard metallicity indicators like the $R_{23}$ index (see, e.g., Kobulnicky \& Kewley 2004) are not accessible to optical telescopes for galaxies at high redshifts. So we estimate the metallicities of galaxies at $1<z<1.5$ from the mass-metallicity relation evolution model by Savaglio et al. (2005), which was established for galaxies at $z<1$. We obtain $12+\log (\mathrm{O} / \mathrm{H})=8.7 \pm 0.2$, i.e., a metallicity close to $Z_{\odot}$. We caveat the reader that metallicity estimates based on nebular lines overestimate the stellar metallicity by up to $60 \%$ (e.g., Bresolin et al. 2005). Nevertheless, a solar-like metallicity is supported by a curve-of-growth analysis 
Table 2. Average properties of the six subsamples defined in Sect. 4.4. The numbers of objects $N$ used for the derivation of the morphological parameters (in parentheses) are smaller than for the complete subsamples, since Hubble ACS images are not available for the K20 quasar field (Sect. 2.2) and some outer parts of the FDF (Sect. 2.1). In the following we list the magnitudes $R$ and $K_{\mathrm{s}}$, the UV-continuum slope parameter $\beta_{\mathrm{b}}$, the UV-bump tracer $\gamma_{34}$, the luminosity at $1500 \AA L_{1500}$, the dust reddening parameter $E(B-V)$, the bolometric luminosity $L_{\text {bol }}$ and the SFR from the model fits, the total stellar mass $M_{\text {stars }}$, the specific SFR $\phi$, the effective radius $R_{\mathrm{e}}$, the Sérsic index $n_{\mathrm{ser}}$, the ellipticity $e$, the CAS parameters concentration $C$ and asymmetry $A$, and the "clumpiness" $R_{\mathrm{T}}+R_{\mathrm{A}}$.

\begin{tabular}{|c|c|c|c|c|c|c|}
\hline Parameter & $\begin{array}{l}1<z<1.5 \\
\beta_{\mathrm{b}}<-1.5\end{array}$ & $\begin{array}{c}1<z<1.5 \\
\beta_{\mathrm{b}}>-1.5 \\
\gamma_{34}>-2\end{array}$ & $\begin{array}{c}1<z<1.5 \\
\beta_{\mathrm{b}}>-1.5 \\
\gamma_{34}<-2\end{array}$ & $\begin{array}{c}2<z<2.5 \\
\beta<-0.4\end{array}$ & $\begin{array}{c}2<z<2.5 \\
\beta>-0.4 \\
\gamma_{34}>-2\end{array}$ & $\begin{array}{c}2<z<2.5 \\
\beta>-0.4 \\
\gamma_{34}<-2 \\
\end{array}$ \\
\hline $\bar{N}$ & $13(12)$ & $14(12)$ & $13(9)$ & 16 & 9 & 9 \\
\hline$R$ [mag] & $23.44 \pm 0.11$ & $23.17 \pm 0.09$ & $23.21 \pm 0.17$ & $23.89 \pm 0.15$ & $23.77 \pm 0.13$ & $23.69 \pm 0.13$ \\
\hline$K_{\mathrm{s}}[\mathrm{mag}]$ & $20.94 \pm 0.24$ & $20.24 \pm 0.11$ & $19.73 \pm 0.09$ & $21.22 \pm 0.18$ & $20.66 \pm 0.22$ & $20.52 \pm 0.20$ \\
\hline$\beta_{\mathrm{b}}$ & $-1.70 \pm 0.06$ & $-0.95 \pm 0.11$ & $-0.72 \pm 0.10$ & $-1.96 \pm 0.09$ & $-1.61 \pm 0.16$ & $-1.37 \pm 0.15$ \\
\hline$\gamma_{34}$ & $-0.44 \pm 0.18$ & $-0.63 \pm 0.29$ & $-3.70 \pm 0.31$ & $+0.36 \pm 0.36$ & $-0.44 \pm 0.35$ & $-3.50 \pm 0.30$ \\
\hline $\log L_{1500}[\mathrm{~W} / \AA ̊ \AA]$ & $33.69 \pm 0.07$ & $33.67 \pm 0.06$ & $33.48 \pm 0.07$ & $34.10 \pm 0.05$ & $34.06 \pm 0.06$ & $34.17 \pm 0.05$ \\
\hline$E_{\mathrm{B}-\mathrm{V}}$ & $0.26 \pm 0.01$ & $0.38 \pm 0.02$ & $0.42 \pm 0.02$ & $0.27 \pm 0.01$ & $0.37 \pm 0.02$ & $0.39 \pm 0.02$ \\
\hline $\log L_{\mathrm{bol}}[\mathrm{W} / \AA ̊ \AA]$ & $11.55 \pm 0.09$ & $12.02 \pm 0.06$ & $11.99 \pm 0.07$ & $11.97 \pm 0.08$ & $12.39 \pm 0.08$ & $12.56 \pm 0.08$ \\
\hline $\log \mathrm{SFR}\left[M_{\odot} / \mathrm{yr}\right]$ & $1.83 \pm 0.08$ & $2.30 \pm 0.08$ & $2.23 \pm 0.09$ & $2.24 \pm 0.09$ & $2.61 \pm 0.08$ & $2.82 \pm 0.08$ \\
\hline $\log M_{\text {stars }}\left[M_{\odot}\right]$ & $9.91 \pm 0.21$ & $10.18 \pm 0.09$ & $10.40 \pm 0.06$ & $10.14 \pm 0.11$ & $10.67 \pm 0.11$ & $10.76 \pm 0.13$ \\
\hline $\log \phi\left[\mathrm{Gyr}^{-1}\right]$ & $0.89 \pm 0.17$ & $1.11 \pm 0.14$ & $0.79 \pm 0.16$ & $1.06 \pm 0.13$ & $0.93 \pm 0.11$ & $1.06 \pm 0.14$ \\
\hline$R_{\mathrm{e}}[\mathrm{kpc}]\left(\right.$ from $\left.\log R_{\mathrm{e}}\right)$ & $2.85 \pm 0.43$ & $3.59 \pm 0.33$ & $3.99 \pm 0.52$ & $2.79 \pm 0.52$ & $3.73 \pm 1.36$ & $3.83 \pm 0.82$ \\
\hline$n_{\mathrm{ser}}$ & $1.32 \pm 0.36$ & $0.98 \pm 0.23$ & $0.54 \pm 0.25$ & $2.07 \pm 0.40$ & $2.48 \pm 0.47$ & $1.61 \pm 0.49$ \\
\hline$e$ & $0.49 \pm 0.07$ & $0.42 \pm 0.06$ & $0.45 \pm 0.07$ & $0.63 \pm 0.04$ & $0.49 \pm 0.07$ & $0.62 \pm 0.07$ \\
\hline$C_{\mathrm{CAS}}$ & $2.43 \pm 0.09$ & $2.16 \pm 0.11$ & $2.07 \pm 0.11$ & $2.52 \pm 0.11$ & $2.46 \pm 0.12$ & $2.19 \pm 0.17$ \\
\hline$A_{\mathrm{CAS}}$ & $0.20 \pm 0.06$ & $0.19 \pm 0.04$ & $0.28 \pm 0.08$ & $0.18 \pm 0.02$ & $0.18 \pm 0.05$ & $0.22 \pm 0.05$ \\
\hline$R_{\mathrm{T}}+R_{\mathrm{A}}$ & $0.24 \pm 0.02$ & $0.24 \pm 0.03$ & $0.26 \pm 0.04$ & $0.25 \pm 0.05$ & $0.40 \pm 0.09$ & $0.32 \pm 0.05$ \\
\hline
\end{tabular}

of interstellar metal absorption lines in $1.3<z<2$ GDDS galaxy spectra (Savaglio et al. 2004).

For galaxies at $z>1.5$, we can use the $J-K$ colour as a proxy for the characteristic age of a galaxy, since $J-K$ brackets the Balmer/4000 $\AA$ break for $2 \leq z \leq 4$. The subsample of FDF galaxies at $2<z<2.5$ exhibits $\langle J-K\rangle=1.7\left(\sigma_{J-K}=0.4\right)$. Hence these galaxies do not meet the $J-K>2.3$ criterion for distant red galaxies at $z>2$ (DRGs, Franx et al. 2003; van Dokkum et al. 2004), so that the bulk of their stellar populations is most probably not much older than $1 \mathrm{Gyr}^{7}$ (see also Pierini et al. 2005). A certain degree of evolution is required by the level of enrichment of the stellar populations reached by galaxies at $2<z<2.5$ in the FDF subsample (see NP05). In fact, for all FDF galaxies at these redshifts with available high$\mathrm{S} / \mathrm{N}$ spectroscopy at rest-frame UV wavelengths, Mehlert et al. (2002, 2006) determined typical metallicities of $0.5-1 Z_{\odot}$, using the stellar-wind feature C IV $\lambda 1550$ and the photometric indices "1370" and "1425" (Leitherer et al. 2001), respectively. This range is in agreement with the mass-metallicity relation for $z \sim 2$ of Erb et al. (2006a).

\subsubsection{No trend between extinction curve and star-formation rate or stellar mass}

Table 2 lists average values of the best-fit SFR obtained for individual galaxies (Sect. 3.2) that are grouped in different subsamples. In general, less reddened galaxies exhibit lower SFRs at any redshift as a consequence of a lower value of the bestfit amount of attenuation. In fact, blue subsamples typically exhibit $E(B-V) \sim 0.2-0.3$, while red subsamples seem to have $E(B-V) \sim 0.4$ on average, whatever the redshift (Table 2).

We note that the SFRs of the $2<z<2.5$ subsamples are generally higher by a factor of 2 to 4 than those of the corresponding

\footnotetext{
7 This age constraint is compatible with the best-fit age estimates of three DRGs $(0.3,1.3$, and $1.4 \mathrm{Gyr})$ of Kriek et al. (2006), based on D4000 indices ranging from 1.2 to 1.4 .
}

$1<z<1.5$ subsamples. The largest differences are observed for red galaxies associated with a significant UV bump. In fact, red galaxies at $2<z<2.5$ tend to exhibit a stronger absorption feature centred at $2175 \AA$ the larger the attenuation, and, thus, the higher the SFR. Consistently, these galaxies are much redder than typical Lyman-break galaxies (cf. Shapley et al. 2004; Noll et al. 2004) and have higher SFRs (about $500 M_{\odot} \mathrm{yr}^{-1}$ on average). Their SFRs exceed those estimated for galaxies selected by UV colour indices (e.g., Erb et al. 2006b) by about one order of magnitude. Daddi et al. (2004) estimated SFRs of almost the same order of magnitude for the K20 galaxies at the highest redshifts in our sample.

In addition, Fig. 12 shows the average total stellar mass as a function of $\gamma_{34}$ for different subsamples. The average total stellar mass is equal to $1.3( \pm 0.3) \times 10^{10} M_{\odot}$ and $2.8( \pm 0.5) \times 10^{10} M_{\odot}$ for galaxies at $1<z<1.5$ and $2<z<2.5$, respectively. It appears to be larger in star-forming galaxies at $1<z<2.5$ that suffer from a larger amount of UV-continuum reddening. However, there is no trend between observed strength of the UV bump and total stellar mass, as already found by NP05.

Finally, we determine specific SFRs, i.e., SFRs per unit of stellar mass. As shown in Table 2, the galaxies in our sample exhibit a narrow range in specific SFR $\left(\phi \sim 10^{1} \mathrm{Gyr}^{-1}\right)$ whatever the redshift. This is a rather large value, especially when considering that $\phi$ has a range of four orders of magnitude for much larger samples that probe the mixture of galaxies at high redshift (e.g., Reddy et al. 2006). A plausible reason for the narrow range of high $\phi$ of our galaxies is that they are all close to the peak of their star-formation history.

\subsubsection{Extinction curve and morphology}

Table 2 lists average values of the effective radius $R_{\mathrm{e}}$, Sérsic index $n_{\text {ser }}$, concentration $C$, asymmetry $A$, and "clumpiness" $R_{\mathrm{T}}+R_{\mathrm{A}}$ of galaxies grouped in the six subsamples that were described previously. We stress that these morphological 

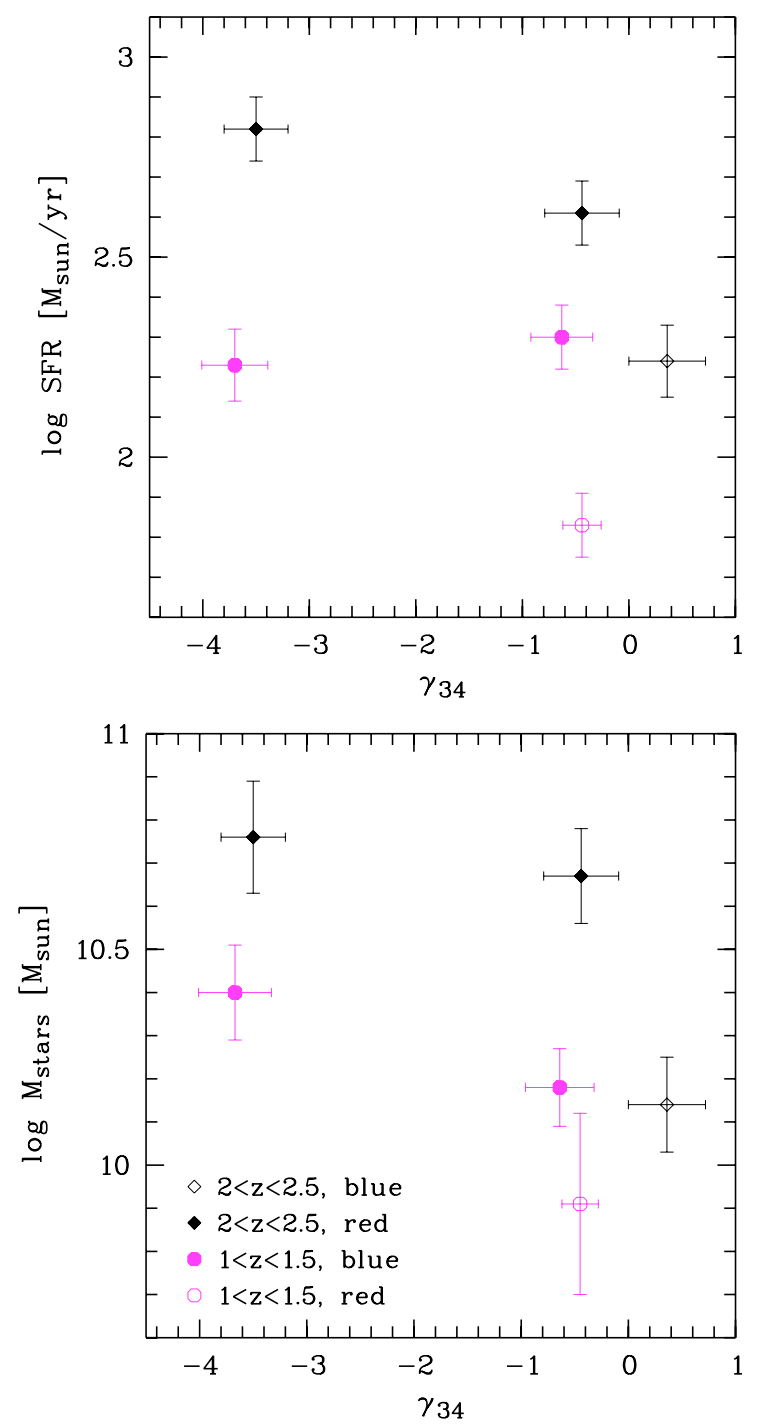

Fig. 12. SFR (top) and total stellar mass (bottom) versus the proxy for the $2175 \AA$ feature $\gamma_{34}$ for the six subsamples defined in Sect. 4.4. Lozenges and circles indicate the FDF $2<z<2.5$ and the FDF+K20 $1<z<1.5$ galaxies, respectively. Open symbols mark the subsamples with low reddening $\left(\beta<-0.4 ; \beta_{\mathrm{b}}<-1.5\right)$, while the highly reddened galaxies are represented by filled symbols. Mean errors are indicated.

parameters were determined in different rest-frame wavelength domains from the mid-UV to the $U$ band depending on redshift (see Sect. 3.4). In general, the rest-frame UV/U-band morphology of a galaxy does not appear to be directly related with the shape of the extinction curve at UV wavelengths whatever the redshift. Nevertheless, blue objects at $2<z<2.5$ and $1<z<1.5$ tend to have smaller effective radii than red objects at the same redshift, whether or not a UV bump is detected in their spectra (see also NP05).

As for the Sérsic index, there is a hint that red galaxies with evidence of a UV bump have a lower $n_{\text {ser }}$ than red galaxies with no evidence of it in both redshift bins (see Fig. 13). For instance, at $1<z<1.5$, the former exhibit an average Sérsic index of $0.54 \pm 0.25$, the latter one equal to $0.98 \pm 0.23$. Overall, the Sérsic index seems to be larger for galaxies at higher redshifts (Fig. 13). Since the available high-resolution imaging probes the rest-frame UV/U-band morphology, the Sérsic profile describes the large-scale distribution of star-formation regions instead of the classical Hubble type. Hence, the
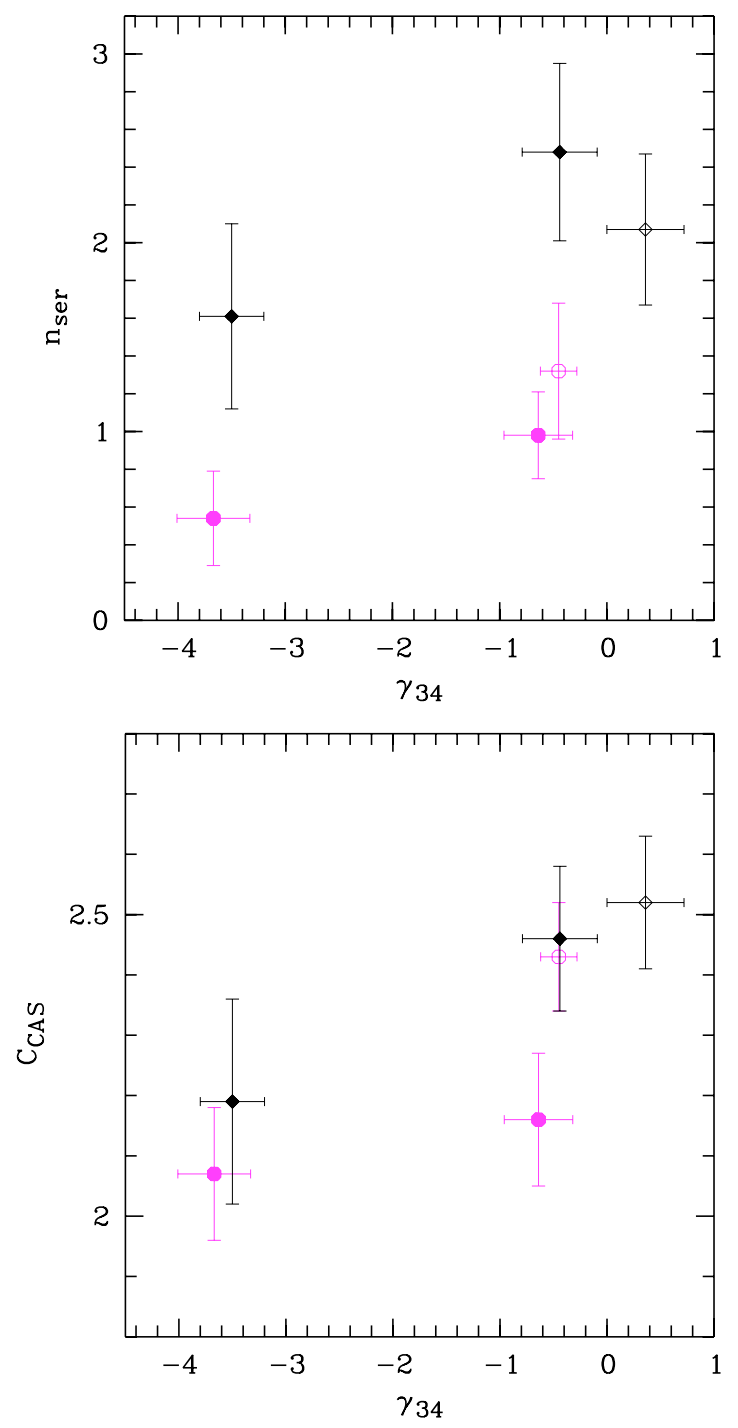

Fig. 13. Sérsic index (top) and concentration (bottom) versus the proxy for the $2175 \AA$ feature $\gamma_{34}$ for the six subsamples defined in Sect. 4.4. Symbols are as in Fig. 12. Mean errors are indicated.

azimuthally-averaged radial distribution of the rest-frame $\mathrm{UV} / U$-band light traced by $n_{\text {ser }}$ is shallower for galaxies with dust producing a significant $2175 \AA$ feature with respect to those without it, whatever the redshift. Furthermore, it is more peaked at higher redshifts than at lower ones, despite this may be an effect of the cosmological brightness dimming. In fact, a faint component of a galaxy like a disc can fail detection more easily at higher redshifts, thus producing a spuriously larger value of $n_{\text {ser. }}$.

In addition to the relatively large effective radii and low Sérsic indices, the low values of concentration of the red galaxies with evidence of a UV bump at $1<z<1.5$ (see Fig. 13) suggest that most of these galaxies are large systems with shallow radial profiles at rest-frame UV/ $U$-band wavelengths. This is not surprising since most of the galaxies at these redshifts can be classified as late types (see Fig. 14). However, the visual inspection of the ACS images reveals that the fraction of objects with a shallow light profile in the rest-frame UV/U-band is $40-70 \%$ if $\beta_{\mathrm{b}}>-1.5$, but only $20-40 \%$ if $\beta_{\mathrm{b}}<-1.5$. For red galaxies with a significant UV bump, this fraction becomes larger (60-80\%), though there are exceptions: CDFS-0271, the 


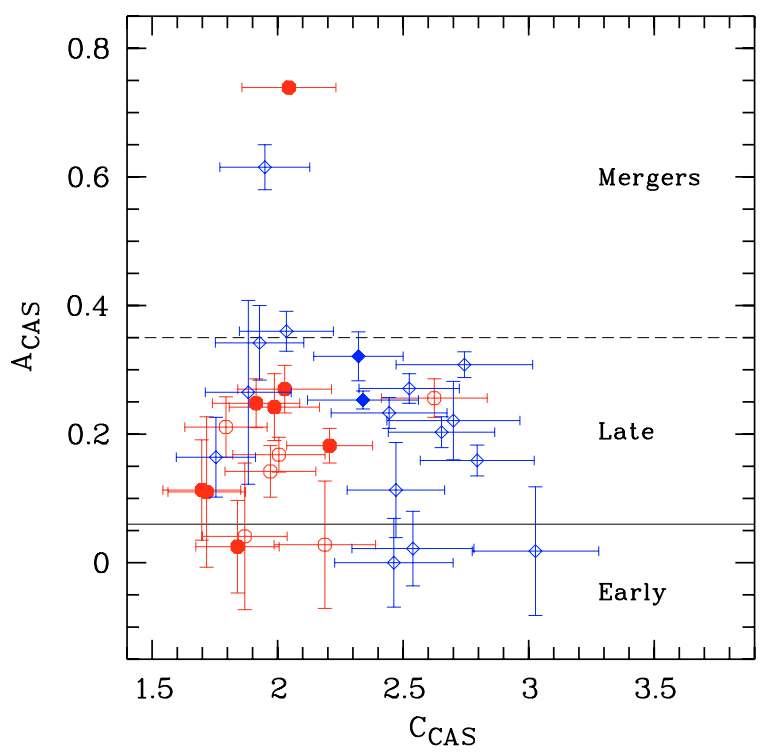

Fig. 14. CAS parameters concentration $C$ and asymmetry $A$ (Conselice et al. 2000, 2003) for our sample of FDF (lozenges) and K20 galaxies (circles) at $1<z<1.5$. Galaxies with $\gamma_{34}<-2$ are marked by filled symbols. The solid and dashed lines separate early-type, late-type, and merging galaxies by their asymmetry (see Conselice et al. 2003).

object with the strongest observed $2175 \AA$ absorption feature, appears as a quite compact galaxy $\left(R_{\mathrm{e}}=2.5 \mathrm{kpc}\right)$.

Establishing the rest-frame UV morphology of a galaxy at $2<z<2.5$ is not possible from the available data, owing to the increased cosmological dimming. Nevertheless, we can investigate the presence of strongly distorted morphologies and/or multiple main components. These characteristics exclude the possibility of a single object with a smooth, radial surface brightness profile in the rest-frame UV. While at $1<z<1.5$ almost all galaxies show only one major component, at $2<z<2.5$ $45-65 \%$ of the objects seem to have two or more main components. There is not much difference between red $(\beta>-0.4)$ and blue galaxies $(\beta<-0.4)$ as for the fraction of objects with multiple components. However, there is a considerable difference when only red objects with strong $2175 \AA$ features are considered. For these galaxies, the fraction of objects with multiple components rises to $70-80 \%$. This suggests that galaxies with extinction curves exhibiting a significant UV bump at $2<z<$ 2.5 are either systems with many, large star-formation complexes or merging systems. This could point to the existence of an intrinsic structural difference with respect to analogous galaxies at $1<z<1.5$, which appear as smooth, disc-like systems in the rest-frame $\mathrm{UV} / U$-band.

\subsection{Properties of the dusty interstellar medium}

For the subsample of galaxies at $2<z<2.5$, the strength of the $2175 \AA$ feature is linked to the average equivalent width $(\mathrm{EW})$ of six prominent, interstellar low-ionisation, absorption lines $W_{\text {LIS }}$ mapped by the FDF spectra (NP05). This relation suggests that the presence of a significant UV bump is linked to high column densities of (cold) gas and dust in the direction towards the observer, since $W_{\text {LIS }}$ traces the covering factor of neutralgas clouds (Shapley et al. 2003). In this scenario, the carriers of the UV bump are protected from strong and hard radiation fields by other (more robust) dust grains (e.g., Gordon et al. 2003). For objects at $1<z<1.5$, there is a lack of strong, purely interstellar absorption lines at the rest-frame UV wavelengths mapped by optical telescopes. Nevertheless, one can investigate the most striking absorption features, i.e., the Fe II doublet at $2600 \AA$ and the $\mathrm{Mg}$ II doublet at $2800 \AA$. We find that the strength of the Fe II absorption does not depend on $\beta_{\mathrm{b}}$ or $\gamma_{34}$, being $W_{\mathrm{Fe} \mathrm{II}} \sim 5 \AA$ for all subsamples. On the other hand, $W_{\text {Mg II }}=1.8 \pm 0.7 \AA$ for blue galaxies but rises to $5.0 \pm 0.5 \AA$ for red galaxies with a strong $2175 \AA$ feature. Hence, the strength of the $\mathrm{Mg}$ II absorption increases together with the apparent strength of the UV bump. The existence of this trend is robust against the contamination of the measured $\mathrm{Mg}$ II equivalent width by an emission contribution from an active galactic nucleus (AGN) since the presence of AGN in the sample is excluded by the line profiles.

The explanation of these results is not straightforward, given the multiple origin of the absorption in the Fe II and particularly the $\mathrm{Mg}$ II doublets. In fact, the latter feature originates in stellar atmospheres as well as in the ISM and, thus, depends on the fraction of intermediate-type stars (particularly A stars), the metallicity and chemical composition of the stellar populations as well as of the ISM, and the structure of the ISM. With these caveats in mind, even a significant contribution of photospheric absorption (see, e.g., Fanelli et al. 1992) to the strength of $\mathrm{Mg}$ II will not harm our viable interpretations, that are based on a purely interstellar origin of the Mg II and Fe II lines. First of all, we note that $\mathrm{Mg}$ and $\mathrm{Fe}$ have similar condensation temperatures and their depletions exhibit a similar systematic trend with density (Whittet 2003). However their relative gas-phase abundances depend on the gas temperature (Savage \& Sembach 1996). In the warmand cold-gas phases, there is less than $10 \%$ and $1 \%$ of Fe, respectively. Furthermore, $\mathrm{Fe}$ is depleted on dust grains about six times more than Mg in the cool ISM, but the Mg-to-Fe gas-phase abundance ratio decreases to about 2 in the warm ISM (Savage \& Sembach 1996). Hence, a larger Mg-to-Fe equivalent width ratio can be explained independently by a greater metallicity (plus depletion on dust grains), as a larger fraction of the ISM being cool or as the ISM being particularly enriched in Mg. Sources of enrichment of the ISM, with a high yield of Mg but only a modest one (if any) of Fe, can be identified as the Asymptotic Giant Branch (AGB) stars. They dominate the overall mass-loss budget at least in the Galaxy (Whittet 2003). Both the presence of these intermediate-mass $\left(2-5 M_{\odot}\right)$ stars and a higher metallicity are naturally consistent with the ages estimated for red galaxies with evidence of a UV bump and a larger Mg-to-Fe equivalent width ratio at $1<z<1.5$ (see Sect. 4.4.1).

\section{Discussion}

In the local Universe, well-characterised extinction curves exist for different environments in the Milky Way and the Magellanic Clouds. From the MW to the SMC, the presence of a broad absorption feature centred at $2175 \AA$ (the UV bump, see Witt $\&$ Lillie 1973, and references therein) vanishes (almost) completely, while the slope of the extinction curve in the far-UV becomes steeper. These differences depend on the mixture of dust grains and molecules present in a system. Tracing them through cosmic times improves our knowledge of the formation and evolution of dust. Furthermore, characterising the typical extinction curves of high-redshift galaxies is crucial for studies of the evolution of the SFR through cosmic times, in particular, if the SFR is determined from the rest-frame UV stellar continuum. Therefore, the number of investigations on the properties of the dusty ISM at intermediate/high redshifts has been increasing in 
recent times (e.g., Malhotra 1997; Pitman et al. 2000; Maiolino et al. 2001; Vernet et al. 2001; Vijh et al. 2003; Hopkins et al. 2004; Savaglio \& Fall 2004; Wang et al. 2004; Wild \& Hewett 2005; NP05; York et al. 2006). In particular, NP05 found that UV-luminous galaxies at $2<z<2.5$ can host dust-producing extinction curves with a significant UV bump. They made use of radiative transfer models with the same dust/stars configuration that helps reproducing properties of dust attenuation in starburst galaxies in the local Universe (Gordon et al. 1997) as well as in the high-redshift one (Vijh et al. 2003). Under this assumption, NP05 concluded that the objects in their sample appear to host a mixture of dust grains that produce extinction curves analogous to those of the SMC and LMC and in between them. Interestingly, Wild \& Hewett (2005) found that damped Ly $\alpha$ absorbers with $0.84<z_{\text {abs }}<1.3$ (identified through $\mathrm{Ca}$ II $(\mathrm{H} \& \mathrm{~K})$ absorption) exhibit extinction curves that are compatible with both the LMC and SMC ones.

A possible connection between these two results is offered by our study. In fact, in the previous sections we have provided a robust evidence for the diversity of the extinction curves characterising 108 massive, UV-luminous galaxies at $1<z<2.5$. Hence, the main conclusion of NP05 is extended down to $z \sim 1$. This evidence stems from the distribution of the data points in the diagnostic diagram portraying the proxy for the strength of the $2175 \AA$ feature $\gamma_{34}$ versus the proxy for reddening in the restframe UV $\beta_{\mathrm{b}}$ (Fig. 5). In addition to the radiative transfer models used by NP05, we make use of additional ones that describe dust attenuation for a disc geometry and include the effect of an age-dependent extinction (Silva et al. 1998; Pierini et al. 2004). This analysis enforces the presence of a considerable fraction of dust out of the disc mid-plane of the sample galaxies, confirming that the dust/stars configuration assumed by NP05 was not too wrong, though simple. The physical foundation of this complex dust/stars configuration is the existence of galactic winds (see, e.g., Murray et al. 2005). More noticeably, the evidence of dust with a UV bump also stems from the direct inspection of the composite spectra obtained for two subsamples of galaxies selected as a function of $\gamma_{34}$ either at $1<z<1.5$ (Fig. 6) or at $2<z<2.5$ (Fig. 11 in NP05).

Our analysis clearly benefits from high $\mathrm{S} / \mathrm{N}$ spectroscopy, whereas others are limited by broad-band photometry. Furthermore, unlike other samples, ours does not suffer from poor statistics and/or the removal of the dominant contribution to the emission by an AGN and/or the potential modification of the dusty ISM by the hard radiation field produced by AGN activity. Finally, it is made of galaxies detected in emission and not in absorption, which can thus be morphologically identified and studied at several wavelengths. These UV-luminous galaxies at $1<z<2.5$ are characterised by large total stellar masses and high SFRs as a consequence of the selection criteria (Sect. 2) ${ }^{8}$. Their stellar masses and SFRs tend to be lower the lower the redshift, consistently with the so-called "downsizing scenario" (Cowie et al. 1996; Gavazzi et al. 1996; Juneau et al. 2005). In fact, this scenario predicts a decrease with cosmic age of the transition mass separating actively from passively-evolving galaxies (e.g., Pannella et al. 2006).

In our sample, galaxies that are heavily reddened in the restframe UV tend to be more massive and have higher SFRs, whatever the redshift. Furthermore, they preferentially exhibit an extinction curve with a significant UV bump. Nevertheless, we do

\footnotetext{
${ }^{8}$ For instance, the need for spectra with a high $\mathrm{S} / \mathrm{N}$ at rest-frame UV wavelengths implies a large fraction of young (i.e., $<100 \mathrm{Myr}$-old) stellar populations.
}

not find any evidence that the observed strength of the UV bump is directly related to either stellar mass or SFR. Clearly these apparently conflicting results call for an interpretation that links properties of the stellar populations to those of the dusty ISM.

For objects at $1<z<1.5$ it is possible to measure the $4000 \AA$ break index D4000 and/or the Balmer-break proxy $C(36-39)$. These indices suggest that dust with a significant UV bump is present in galaxies where intermediate-age (i.e., from 0.2 to 1-2 Gyr-old) stellar populations exist and/or that are about three times older than those without a UV bump detection (see Sect. 4.4.1). The existence of a link between extinction curve and the characteristic age of the stellar populations of a galaxy is nicely supported by the analysis of the absorption-line ratios that are available for the same objects. In fact, we find that the ratio of the absorption strengths in the Mg II doublet at $2800 \AA$ and in the Fe II doublet at $2600 \AA$ is larger in more UV-reddened galaxies with evidence of a UV bump. This holds whether the absorption arises in the ISM or in stellar atmospheres. Finally, galaxies with extinction curves exhibiting a significant UV bump at $1<z<1.5$ predominantly appear as ordinary disc systems, at variance with galaxies without a UV-bump detection at similar redshifts, which appear as irregular systems. Smoother morphologies are also consistent with a relatively longer starformation history.

In general, models with a mixed dust/stars configuration where MW-type dust is distributed in a geometrically thin disc (e.g., Silva et al. 1998; Pierini et al. 2004) can account for values of $-2.5<\gamma_{34}<1$ in our sample of $1<z<1.5$ galaxies (see Fig. 9). However, they predict a rapid decrease of the observed strength of the UV bump as soon as the attenuation at UV wavelengths becomes large along the line of sight. In order to describe the increase of the observed strength of the UV bump with increasing reddening and the existence of galaxies with $\gamma_{34}<-3$, a screen-like geometry needs to be invoked (e.g., Witt \& Gordon 2000). The presence of large amounts of dust above the disc mid-plane can be obtained in principle via galactic winds, starburst superwinds, and even AGN activity. It is favoured by the presence of magnetic fields (e.g., Greenberg et al. 1987). On the other hand, the ISM of objects with $\gamma_{34} \sim 1$ does not seem to contain a significant fraction of carriers of the UV bump. The dust attenuation properties of these galaxies are similar to those of nearby starbursts (e.g., Calzetti et al. 1994). Consistently, our $1<z<1.5$ galaxies without a detection of the UV bump tend to be dominated by young (i.e., $\$ 100 \mathrm{Myr}$-old) stellar populations and to have irregular morphologies.

There is no spectral indication for the characteristic ages of the stellar populations in the UV-bright galaxies at $2<z<2.5$. However, the mean $J-K$ colour of the subsample of FDF galaxies at these redshifts $(\langle J-K\rangle=1.7)$ suggests that the bulk of their stellar populations is most probably not much older than 1 Gyr (Franx et al. 2003; van Dokkum et al. 2004; Pierini et al. 2005). Furthermore, their metallicities appear to be about solar, as well as for the galaxies at $1<z<1.5$ (see Sect. 4.4.1).

On the other hand, the subsample of UV-bright galaxies at $2<z<2.5$ offers the possibility to probe the ISM topology from the determination of the EWs of strong interstellar absorption lines (cf. Shapley et al. 2003). For these objects, NP05 concluded that the presence of the UV bump does not seem to depend on the total metallicity, as given by the EW of the C IV doublet at $1550 \AA$. Conversely, it seems to be associated with a large average EW of the six most prominent interstellar low-ionisation absorption lines in the far-UV. NP05 interpreted this result as indication of a larger covering fraction of young 
massive stars by neutral gas clouds (containing dust grains).

At this point, it is possible to piece together the individual clues on the origin of the different extinction curves of UV-luminous galaxies at $1<z<2.5$ and formulate a selfconsistent scenario. In particular, we can address physical reasons for the presence or absence of the UV bump. First of all, we recall that the nature of the carriers of the $2175 \AA$ feature is probably multiple, i.e., organic carbon (as in clusters of polycyclic aromatic hydrocarbons, PAHs) and amorphous silicates (Bradley et al. 2005, and references therein). As supernovae $(\mathrm{SNe})$ manufacture many of the condensible elements, clearly they are candidate sources of dust. In particular, the expanding envelope of a type-II SN may represent an O-rich environment where silicate dust can form. Silicon carbide and amorphous carbon are the main products of $\mathrm{C}$-rich stars of intermediate mass (Whittet 2003). Conversely, intermediate-mass, O-rich stars produce silicate dust. These stars lose mass copiously during the red giant and asymptotic giant branch phases of their evolution. As seen before, AGB stars (with ages from 0.2 to $1-2 \mathrm{Gyr}$ ) are most probably present in large numbers in galaxies which exhibit the $2175 \AA$ absorption feature in their spectra. These stars are considered to be the main source of the probable carriers of the UV bump also in the local Universe (e.g., Galliano 2006).

Once the basic units are produced and injected into the general ISM, dust reprocessing takes place there. Our study seems to confirm that dust self-shielding is important for the survival of the carriers of the UV bump in the general ISM (cf. Gordon et al. 2003), despite other processes may modify or destroy them (see Whittet et al. 2004). This is consistent with recent studies on the formation and survival of PAH clusters in photodissociation regions (Rapacioli et al. 2006, and references therein). Milder UV radiation fields, as found in galaxies at $1<z<1.5$, ease the need for self-shielding of course. An extreme example of this is the Milky Way, which has a relatively quiescent star-formation activity, a metal-enriched and aged ISM, and an average extinction curve with a very pronounced UV bump (e.g., Cardelli et al. 1989; see also Fitzpatrick \& Massa 2007 for a recent analysis of the shapes of MW interstellar extinction curves). We recall that galaxies hosting dust with a significant UV bump at $1<z<1.5$ exhibit the heaviest UV reddening. In our preferred interpretation, this is at least partly due to the screen-like effect of dust lifted from the disc mid-plane by galactic winds.

Establishing the presence of different mixtures of dust grains in the dusty interstellar media of different high-redshift galaxies has important consequences on the perception of the cosmological evolution of the SFR. As shown in Fig. 15, attenuation models where all stars are enshrouded by dust predict corrections of the luminosity at $1500 \AA$ (rest-frame) that differ by up to $\sim 40 \%$ for the LMC and SMC extinction curves at fixed attenuation in the $V$-band (rest-frame) $A_{\mathrm{V}}<2$. Furthermore, a Calzetti law systematically overpredicts the correction of $L_{1500}$ and, thus, of the SFR with respect to an attenuation model with LMC-type dust. This is also true with respect to an attenuation model with SMC-type dust, the overestimate being much larger than $40 \%$ when $A_{\mathrm{V}}>1$. In conclusion, not understanding amount, composition, topology, and three-dimensional distribution of dust in a galaxy can seriously undermine our knowledge of galaxy evolution.

\section{Conclusions}

High signal-to-noise optical spectra available for 108 massive, UV-luminous galaxies at $1<z<2.5$ allow to constrain properties of the extinction curve at rest-frame UV wavelengths as

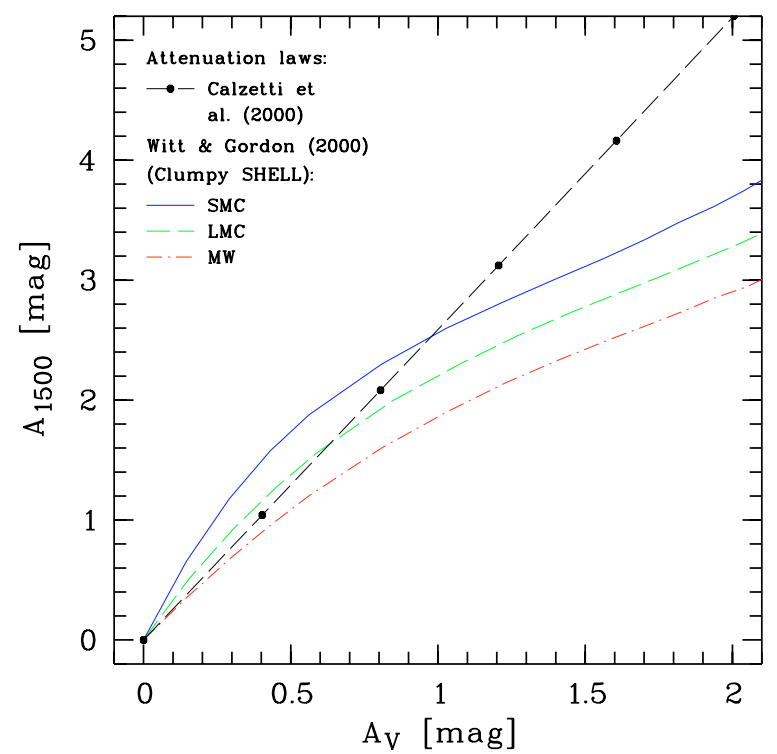

Fig. 15. Attenuation at $1500 \AA$ versus attenuation in the $V$ band for three radiative transfer models of Witt \& Gordon (2000) with clumpy SHELL dust geometry and the empirical Calzetti et al. (2000) law (see legend and Fig. 3). In the latter case filled circles are plotted in intervals of $\Delta E(B-V)=0.1$.

discussed by Noll \& Pierini (2005, NP05). As a main result, we find that these galaxies host different mixtures of dust grains and molecules, which produce different extinction curves. In particular, the majority of the rest-frame UV spectral energy distributions (SEDs) suffering from heavy reddening exhibit a broad absorption excess centred at $2175 \AA$ (the so-called "UV bump"). Hence the associated galaxies host dust that can produce an extinction curve similar to the average one determined for the interstellar medium (ISM) of the Large Magellanic Cloud or even of the Milky Way. Conversely, the extinction curve of galaxies with the least reddened UV SEDs is consistent with the average, featureless, and steep extinction curve of the Small Magellanic Cloud, if the strength of the apparent UV bump is not considerably reduced by dust-stars mixing and age-dependent extinction effects as suggested for local disc galaxies.

For objects at $1<z<1.5$, the comparison of measured and synthetic stellar spectral indices shows that dust with a significant UV bump is present in galaxies that host a rather large fraction of intermediate-age stars (i.e., from 0.2 to $1-2$ Gyr old) at these redshifts. The older ages and the higher reddening at UV wavelengths found for these galaxies are consistent with the larger Mg II-to-Fe II ratio that we measure. At the same time, older ages are consistent with a larger fraction of smooth, radial surface brightness profiles in the rest-frame optical. Therefore, at $1<z<1.5$ galaxies with evidence of a UV bump appear as disc systems which are more evolved than the more compact, clumpy objects whose dust properties resemble those of nearby starburst galaxies. Nevertheless, part of the dusty ISM seems to be lifted above the disc mid-plane owing to the action of galactic winds.

In a complementary way, at least for objects at $2<z<2.5$, the equivalent width of the most prominent interstellar absorption lines in the far-UV carries information on the topology of the ISM. As NP05 showed, the covering fraction of young massive stars by neutral gas clouds (containing dust) is larger in galaxies with evidence of a UV bump than in those with an undetected $2175 \AA$ feature. 
The previous results can be interpreted in a self-consistent way by a scenario where the basic units of the (probably multiple) carriers of the UV bump mostly originate from intermediate-mass $\left(2-5 M_{\odot}\right)$ stars in late evolution phases. A high production rate of the carriers is, therefore, connected to high amounts of the required stars, which needs suitable star-formation histories. The carriers of the $2175 \AA$ feature are most probably made of organic carbon and amorphous silicates. They can survive in the harsh environments of UV-luminous high-redshift galaxies owing to dust self-shielding, which is most efficient in massive, gas-rich, high-metallicity galaxies. As indicated by our analysis, good candidates for galaxies with a pronounced UV bump in their extinction curves (obviously fulfilling the requirements above) are highly dust-reddened, UVbright galaxies at $2<z<2.5$ and near-IR bright, intensely star-forming galaxies at $1<z<1.5$.

The existence of different extinction curves implies that different patterns of evolution and reprocessing of dust exist among massive, UV-luminous galaxies at high redshift. Ignoring this may produce a non-negligible uncertainty on the SFR estimate of a galaxy based on the rest-frame UV.

Acknowledgements. We thank Karl D. Gordon for providing dust attenuation models with LMC-type dust. We also wish to thank Anna Gallazzi and Adolf N. Witt for useful discussions and suggestions. MP thanks Chris Conselice for making available the IRAF code of the CAS algorithm. Finally, we thank the anonymous referee for her/his helpful comments that improved this paper. The spectra of the FDF and K20 samples are based on observations obtained with FORS at the ESO VLT, Paranal, Chile. The latter spectra as well as the Hubble ACS images of the CDFS were retrieved from the ESO/ST-ECF Science Archive Facility. The GDDS spectra stem from observations with GMOS at the Gemini North Telescope, Mauna Kea, USA. The investigation of our sample of local starburst galaxies has been based on INES data from the IUE satellite and has made use of the NASA/IPAC Extragalactic Database (NED) which is operated by the Jet Propulsion Laboratory, California Institute of Technology, under contract with the National Aeronautics and Space Administration. This research was supported by the German Science Foundation (DFG, SFB 375).

\section{References}

Abraham, R. G., Valdes, F., Yee, H. K. C., \& van den Bergh, S. 1994, ApJ, 432, 75

Abraham, R. G., Tanvir, N. R., Santiago, B. X., et al. 1996, MNRAS, 279, L47 Abraham, R. G., Glazebrook, K., McCarthy, P. J., et al. 2004, AJ, 127, 2455

Balogh, M. L., Morris, S. L., Yee, H. K. C., et al. 1999, ApJ, 527, 54

Bertin, E., \& Arnouts, S. 1996, A\&AS, 117, 393

Bradley, J., Dai, Z. R., Erni, R., et al. 2005, Science, 307, 244

Bresolin, F., Schaerer, D., González Delgado, R. M., \& Stasińska, G. 2005, A\&A, 441, 981

Brinchmann, J., \& Ellis, R. S. 2000, ApJ, 536, L77

Calzetti, D., Kinney, A. L., \& Storchi-Bergmann, T. 1994, ApJ, 429, 582

Calzetti, D., Armus, L., Bohlin, R. C., et al. 2000, ApJ, 533, 682

Cardelli, J. A., Clayton, G. C., \& Mathis, J. S. 1989, ApJ, 345, 245

Cimatti, A., Mignoli, M., Daddi, E., et al. 2002, A\&A, 392, 395

Clayton, G. C. 2004, in Astrophysics of dust, ed. A. N. Witt, G. C. Clayton, \& B. T. Draine, ASP Conf. Ser., 309, 57

Conselice, C. J., Bershady, M. A., \& Jangren, A. 2000, ApJ, 529, 886

Conselice, C. J., Bershady, M. A., Dickinson, M., \& Papovich, C. 2003, AJ, 126, 1183

Cowie, L. L., Songaila, A., Hu, E. M., \& Cohen, J. G. 1996, AJ, 112, 839

Daddi, E., Cimatti, A., Renzini, A., et al. 2004, ApJ, 600, L127

Drory, N., Salvato, M., Gabasch, A., et al. 2005, ApJ, 619, L131

Elmegreen, B. G., Elmegreen, D. M., \& Montenegro, L. 1992, ApJS, 79, 37

Erb, D. K., Shapley, A. E., Pettini, M., et al. 2006a, ApJ, 644, 813

Erb, D. K., Steidel, C. C., Shapley, A. E., et al. 2006b, ApJ, 647, 128

Fanelli, M. N., O'Connell, R. W., Burstein, D., \& Wu, C. C. 1992, ApJS, 82, 197

Fernandes, R. C., Mateus, A., Sodré, L., Stasińska, G., \& Gomes, J. M. 2005, MNRAS, 358, 363

Fischera, J., Dopita, M. A., \& Sutherland, R. S. 2003, ApJ, 599, L21

Fitzpatrick, E. L. 2004, in Astrophysics of dust, ed. A. N. Witt, G. C. Clayton, \& B. T. Draine, ASP Conf. Ser., 309, 33
Fitzpatrick, E. L., \& Massa, D. 2007, [arXiv:0705. 0154] Fontana, A., Donnarumma, I., Vanzella, E., et al. 2003, ApJ, 594, L9 Franx, M., Labbé, I., Rudnick, G., et al. 2003, ApJ, 587, L79 Gabasch, A., Bender, R., Seitz, S., et al. 2004, A\&A, 421, 41 Galliano, F. 2006 [arXiv: astro-ph/0610852]

Gavazzi, G., Pierini, D., \& Boselli, A. 1996, A\&A, 312, 397

Giavalisco, M., Ferguson, H. C., Koekemoer, A. M., et al. 2004, ApJ, 600, L93 Glazebrook, K., Abraham, R. G., McCarthy, P. J., et al. 2004, Nature, 430, 181 Gordon, K. D., Calzetti, D., \& Witt, A. N. 1997, ApJ, 487, 625

Gordon, K. D., Clayton, G. C., Misselt, K. A., Landolt, A. U., \& Wolff, M. J. 2003, ApJ, 594, 279

Greenberg, J. M., Ferrini, F., Barsella, B., \& Aiello, S. 1987, Nature, 327, 214 Hamilton, D. 1985, ApJ, 297, 371

Heidt, J., Appenzeller, I., Gabasch, A., et al. 2003, A\&A, 398, 49

Hopkins, P. F., Strauss, M. A., Hall, P. B., et al. 2004, AJ, 128, 1112

Inoue, A. K., Buat, V., Burgarella, D., et al. 2006, MNRAS, 370, 380

Juneau, S., Glazebrook, K., Crampton, D., et al. 2005, ApJ, 619, 135

Kauffmann, G., Heckman, T. M., White, S. D. M., et al. 2003, MNRAS, 341, 33 Kennicutt, R. C. Jr. 1998, ARA\&A, 36, 189

Kewley, L. J., Geller, M. J., \& Jansen, R. A. 2004, AJ, 127, 2002

Kobulnicky, H. A., \& Kewley, L. J. 2004, ApJ, 617, 240

Kriek, M., van Dokkum, P. G., Franx, M., et al. 2006, ApJ, 645, 44

Le Borgne, D., Abraham, R., Daniel, K., et al. 2006, ApJ, 642, 48

Leitherer, C., Leão, J. R. S., Heckman, T. M., et al. 2001, ApJ, 550, 724

Leitherer, C., Calzetti, D., \& Martins, L. P. 2002a, ApJ, 574, 114

Leitherer, C., Li, I.-H., Calzetti, D., \& Heckman, T. M. 2002b, ApJS, 140, 303

Maiolino, R., Marconi, A., Salvati, M., et al. 2001, A\&A, 365, 28

Malhotra, S. 1997, ApJ, 488, L101

Maraston, C. 2005, MNRAS, 362, 799

Mehlert, D., Noll, S., Appenzeller, I., et al. 2002, A\&A, 393, 809

Mehlert, D., Tapken, C., Appenzeller, I., et al. 2006, A\&A, 455, 835

Menanteau, F., Ford, H. C., Motta, V., et al. 2006, AJ, 131, 208

Mignoli, M., Cimatti, A., Zamorani, G., et al. 2005, A\&A, 437, 883

Murray, N., Quataert, E., \& Thompson, T. A. 2005, ApJ, 618, 569

Mutchler, M., Koekemoer, A. M., \& Hack, W. 2002, in The 2002 HST Calibration Workshop: Hubble after the Installation of the ACS and the NICMOS Cooling System, ed. S. Arribas, A. Koekemoer, \& B. Whitmore, 70

Noll, S., \& Pierini, D. 2005, A\&A, 444, 137 (NP05)

Noll, S., Mehlert, D., Appenzeller, I., et al. 2004, A\&A, 418, 885

Pannella, M., Hopp, U., Saglia, R. P., et al. 2006, ApJ, 639, L1

Panuzzo, P., Granato, G. L., Buat, V., et al. 2007, MNRAS, 375, 640

Pierini, D., Gordon, K. D., Witt, A. N., \& Madsen, G. J. 2004, ApJ, 617, 1022

Pierini, D., Maraston, C., Gordon, K. D., \& Witt, A. N. 2005, MNRAS, 363, 131

Pitman, K. M., Clayton, G. C., \& Gordon, K. D. 2000, PASP, 112, 537

Rapacioli, M., Calvo, F., Joblin, C., et al. 2006, A\&A, 460, 519

Reddy, N. A., Steidel, C. C., Fadda, D., et al. 2006, ApJ, 644, 792

Salpeter, E. E. 1955, ApJ, 121, 161

Savage, B. D., \& Sembach, K. R. 1996, ARA\&A, 34, 279

Savaglio, S., \& Fall, S. M. 2004, ApJ, 614, 293

Savaglio, S., Glazebrook, K., Abraham, R. G., et al. 2004, ApJ, 602, 51

Savaglio, S., Glazebrook, K., Le Borgne, D., et al. 2005, ApJ, 635, 260

Schade, D., Lilly, S. J., Crampton, D., et al. 1995, ApJ, 451, L1

Schlegel, D. J., Finkbeiner, D. P., \& Davis, M. 1998, ApJ, 500, 525

Sérsic, J. L. 1968, Atlas de galaxias australes (Cordoba, Observatorio Astronomico)

Shapley, A. E., Steidel, C. C., Pettini, M., \& Adelberger, K. L. 2003, ApJ, 588, 65

Shapley, A. E., Erb, D. K., Pettini, M., Steidel, C. C., \& Adelberger, K. L. 2004, ApJ, 612, 108

Silva, L., Granato, G. L., Bressan, A., \& Danese, L. 1998, ApJ, 509, 103

Simard, L., Koo, D. C., Faber, S. M., et al. 1999, ApJ, 519, 563

Tuffs, R. J., Popescu, C. C., Völk, H. J., et al. 2004, A\&A, 419, 821

van Dokkum, P. G., Franx, M., Förster Schreiber, N. M., et al. 2004, ApJ, 611, 703

Vernet, J., Fosbury, R. A. E., Villar-Martín, M., et al. 2001, A\&A, 366, 7

Vijh, U. P., Witt, A. N., \& Gordon, K. D. 2003, ApJ, 587, 533

Wang, J., Hall, P. B., Ge, J., Li, A., \& Schneider, D. P. 2004, ApJ, 609, 589

Whittet, D. C. B. 2003, Dust in the galactic environment, 2nd Ed. (Bristol: Institute of Physics (IOP) Publishing)

Whittet, D. C. B., Shenoy, S. S., Clayton, G. C., \& Gordon, K. D. 2004, ApJ, 602,291

Wild, V., \& Hewett, P. C. 2005, MNRAS, 361, L30

Witt, A. N., \& Lillie, C. F. 1973, A\&A, 25, 397

Witt, A. N., \& Gordon, K. D. 2000, ApJ, 528, 799

Wu, K. L.-K. 1999, Ph.D. Thesis, University of California, Santa Cruz

York, D. G., Khare, P., Vanden Berk, D., et al. 2006, MNRAS, 367, 945 


\section{Online Material}


Table 1. Basic parameters, i.e., ID, world coordinates (J2000), $R$ and $K_{\mathrm{s}}$ magnitudes, and redshift, of the 108 sample galaxies from the FDF, K20, and GDDS. For the origin of the data see Sect. 2. Furthermore, the main parameters of this study, $\beta_{\mathrm{b}}$ and $\gamma_{34}$ (see Sect. 3.1), are listed. Due to non-optimal wavelength ranges, $\beta_{\mathrm{b}}$ could only be measured for 88 objects.

\begin{tabular}{|c|c|c|c|c|c|c|c|}
\hline ID & RA & Dec & $R$ & $K_{\mathrm{s}}$ & $z$ & $\beta_{\mathrm{b}}$ & $\gamma_{34}$ \\
\hline CDFS-0047 & 033216.35 & -274823.9 & 23.43 & 19.34 & 1.295 & $-0.37 \pm 0.22$ & $-4.43 \pm 0.34$ \\
\hline CDFS-0112 & 033237.63 & -274744.0 & 23.00 & 19.20 & 1.097 & $-1.20 \pm 0.10$ & $-0.83 \pm 0.11$ \\
\hline CDFS-0154 & 033221.75 & -274724.6 & 23.77 & 19.68 & 1.000 & $-0.37 \pm 0.26$ & $-4.99 \pm 0.32$ \\
\hline CDFS-0201 & 033210.57 & -274706.2 & 22.92 & 19.24 & 1.045 & $-0.90 \pm 0.10$ & $-1.29 \pm 0.16$ \\
\hline CDFS-0217 & 033230.72 & -274617.2 & 23.42 & 19.25 & 1.307 & $-0.19 \pm 0.21$ & $-3.69 \pm 0.27$ \\
\hline CDFS-0226 & 033211.57 & -274622.9 & 22.34 & 19.35 & 1.221 & $-1.22 \pm 0.11$ & $-0.85 \pm 0.11$ \\
\hline CDFS-0239 & 033229.09 & -274629.0 & 23.11 & 19.45 & 2.227 & $-2.37 \pm 0.21$ & $-0.67 \pm 0.22$ \\
\hline CDFS-0246 & 033236.45 & -274631.6 & 23.04 & 19.15 & 1.047 & $+9.99 \pm 9.99$ & $-0.65 \pm 0.28$ \\
\hline CDFS-0254 & 033208.89 & -274629.1 & 24.29 & 19.82 & 1.032 & $+9.99 \pm 9.99$ & $-4.75 \pm 0.41$ \\
\hline CDFS-0271 & 033234.86 & -274640.4 & 23.84 & 19.37 & 1.097 & $-0.50 \pm 0.19$ & $-7.24 \pm 0.31$ \\
\hline CDFS-0295 & 033237.36 & -274645.5 & 23.53 & 19.88 & 1.843 & $+9.99 \pm 9.99$ & $-5.25 \pm 0.13$ \\
\hline CDFS-0310 & 033219.34 & -274701.1 & 23.89 & 19.14 & 1.166 & $-0.48 \pm 0.11$ & $-1.21 \pm 0.16$ \\
\hline CDFS-0331 & 033224.64 & -274407.8 & 22.85 & 19.68 & 1.901 & $-1.31 \pm 0.12$ & $+1.63 \pm 0.11$ \\
\hline CDFS-0344 & 033237.41 & -274407.0 & 23.43 & 18.86 & 1.017 & $+9.99 \pm 9.99$ & $-3.15 \pm 0.30$ \\
\hline CDFS-0346 & 033223.71 & -274411.8 & 23.38 & 19.31 & 2.060 & $-0.82 \pm 0.09$ & $-1.10 \pm 0.13$ \\
\hline CDFS-0384 & 033235.34 & -274419.3 & 23.27 & 19.87 & 1.224 & $-0.94 \pm 0.13$ & $-2.61 \pm 0.16$ \\
\hline CDFS-0407 & 033217.96 & -274431.4 & 23.21 & 19.50 & 1.227 & $-2.02 \pm 0.12$ & $-1.89 \pm 0.23$ \\
\hline CDFS-0413 & 033236.03 & -274423.8 & 22.47 & 19.16 & 1.038 & $-1.41 \pm 0.05$ & $-4.24 \pm 0.07$ \\
\hline CDFS-0416 & 033235.13 & -274439.6 & 23.22 & 19.58 & 1.038 & $+0.20 \pm 0.23$ & $-6.91 \pm 0.31$ \\
\hline CDFS-0522 & 033229.12 & -274521.1 & 24.32 & 19.94 & 2.226 & $-1.37 \pm 0.23$ & $-0.57 \pm 0.30$ \\
\hline CDFS-0525 & 033212.47 & -274530.3 & 23.32 & 19.58 & 1.375 & $-2.41 \pm 0.17$ & $-3.31 \pm 0.19$ \\
\hline CDFS-0582 & 033212.20 & -274554.4 & 22.48 & 19.20 & 1.038 & $-0.94 \pm 0.20$ & $-1.43 \pm 0.18$ \\
\hline CDFS-0587 & 033223.09 & -274558.1 & 23.35 & 19.99 & 1.727 & $-1.51 \pm 0.06$ & $-1.15 \pm 0.11$ \\
\hline CDFS-0693 & 033211.00 & -274459.4 & 23.47 & 19.73 & 1.553 & $-0.65 \pm 0.10$ & $-0.13 \pm 0.24$ \\
\hline FDF-1208 & 010551.87 & -254803.5 & 24.01 & 20.47 & 2.178 & $-1.46 \pm 0.14$ & $+0.06 \pm 0.39$ \\
\hline FDF-1473 & 010552.93 & -254519.2 & 23.59 & 22.74 & 1.580 & $-1.72 \pm 0.07$ & $+1.12 \pm 0.13$ \\
\hline FDF-1496 & 010553.02 & -254803.9 & 23.28 & 20.24 & 1.376 & $-0.44 \pm 0.10$ & $-1.74 \pm 0.17$ \\
\hline FDF-1691 & 010553.71 & -254528.7 & 24.16 & 21.78 & 2.344 & $-0.85 \pm 0.16$ & $-0.60 \pm 0.23$ \\
\hline FDF-1744 & 010553.90 & -254606.3 & 24.35 & 21.43 & 2.374 & $-2.54 \pm 0.21$ & $+2.42 \pm 0.41$ \\
\hline FDF-1823 & 010554.18 & -254330.5 & 22.89 & 19.80 & 1.050 & $-1.64 \pm 0.10$ & $-2.39 \pm 0.11$ \\
\hline FDF-1922 & 010554.58 & -254700.1 & 23.53 & 22.32 & 1.827 & $-2.15 \pm 0.10$ & $+0.54 \pm 0.12$ \\
\hline FDF-1991 & 010554.83 & -254616.3 & 24.64 & 21.46 & 2.334 & $-2.46 \pm 0.41$ & $9 \pm 0.79$ \\
\hline FDF-2274 & 010555.89 & -254434.1 & 23.57 & 20.92 & 2.253 & $-1.59 \pm 0.10$ & $+0.42 \pm 0.40$ \\
\hline FDF-2295 & 010555.98 & -254312.3 & 23.17 & 20.01 & 1.824 & $-1.62 \pm 0.12$ & $+0.06 \pm 0.27$ \\
\hline FDF-2334 & 010556.13 & -254439.9 & 23.34 & 20.98 & 1.392 & $-1.09 \pm 0.07$ & $-0.33 \pm 0.08$ \\
\hline FDF-2418 & 010556.44 & -254512.1 & 23.38 & 19.72 & 2.332 & $-2.06 \pm 0.20$ & $-1.94 \pm 0.22$ \\
\hline FDF-2495 & 010556.75 & -254343.9 & 23.59 & 20.95 & 2.453 & $-1.15 \pm 0.09$ & $-2.51 \pm 0.69$ \\
\hline FDF-2636 & 010557.31 & -254402.2 & 23.79 & 20.05 & 2.253 & $-0.87 \pm 0.12$ & $-4.49 \pm 0.71$ \\
\hline FDF-3005 & 010558.61 & -254814.1 & 23.79 & 20.08 & 2.253 & $-1.59 \pm 0.15$ & $-1.69 \pm 0.29$ \\
\hline FDF-3163 & 010559.17 & -254538.1 & 23.55 & 20.25 & 2.441 & $-1.00 \pm 0.16$ & $-2.27 \pm 0.82$ \\
\hline FDF-3230 & 010559.40 & -254407.6 & 22.72 & 18.46 & 1.154 & $+9.99 \pm 9.99$ & $-3.07 \pm 0.72$ \\
\hline FDF-3300 & 010559.64 & -254629.9 & 24.09 & 21.53 & 2.375 & $-2.37 \pm 0.32$ & $+1.19 \pm 0.64$ \\
\hline FDF-3374 & 010559.88 & -254510.7 & 23.57 & 20.22 & 2.386 & $-1.68 \pm 0.13$ & $-0.21 \pm 0.29$ \\
\hline FDF-3476 & 010600.24 & -254455.7 & 23.20 & 20.62 & 1.438 & $-1.53 \pm 0.05$ & $+0.08 \pm 0.07$ \\
\hline FDF-3688 & 010600.89 & -254705.3 & 24.25 & 21.36 & 2.375 & $-2.36 \pm 0.27$ & $-0.58 \pm 0.61$ \\
\hline FDF-3781 & 010601.22 & -254622.2 & 23.68 & 21.30 & 1.027 & $-1.59 \pm 0.21$ & \pm 0.61 \\
\hline FDF-3810 & 010601.32 & -254527.9 & 22.95 & 19.74 & 2.372 & $-1.10 \pm 0.13$ & $-3.24 \pm 0.25$ \\
\hline FDF-3874 & 010601.51 & -254546.4 & 23.54 & 20.12 & 2.483 & $-2.27 \pm 0.18$ & $-2.73 \pm 1.00$ \\
\hline FDF-3875 & 010601.51 & -254733.6 & 24.64 & 22.02 & 2.243 & $-1.91 \pm 0.13$ & $-1.14 \pm 0.35$ \\
\hline FDF-3958 & 010601.84 & -254428.8 & 23.99 & 21.69 & 2.130 & $-2.42 \pm 0.21$ & $+1.09 \pm 0.30$ \\
\hline FDF-4049 & 010602.15 & -254717.5 & 23.51 & 20.26 & 1.475 & $-1.11 \pm 0.05$ & $-2.99 \pm 0.07$ \\
\hline FDF-4092 & 010602.33 & -254329.5 & 23.77 & 22.80 & 1.394 & $-1.58 \pm 0.09$ & $+0.00 \pm 0.11$ \\
\hline FDF-4115 & 010602.38 & -254724.8 & 21.87 & 19.09 & 1.317 & $-1.67 \pm 0.08$ & $+0.12 \pm 0.08$ \\
\hline FDF-4310 & 010603.03 & -254356.9 & 23.76 & 21.30 & 1.667 & $-1.66 \pm 0.15$ & $+0.39 \pm 0.20$ \\
\hline FDF-4324 & 010603.09 & -254357.8 & 23.65 & 20.60 & 1.667 & $-1.39 \pm 0.14$ & $+0.76 \pm 0.24$ \\
\hline FDF-4479 & 010603.70 & -254453.5 & 23.56 & 20.79 & 1.304 & $-1.02 \pm 0.07$ & $-1.09 \pm 0.12$ \\
\hline FDF-4795 & 010604.81 & -254713.9 & 23.66 & 20.78 & 2.159 & $-1.95 \pm 0.18$ & $+0.05 \pm 0.18$ \\
\hline FDF-4871 & 010605.06 & -254603.5 & 23.53 & 20.53 & 2.472 & $-1.22 \pm 0.21$ & $-3.89 \pm 0.50$ \\
\hline FDF-4996 & 010605.50 & -254627.8 & 23.45 & 20.91 & 2.028 & $-1.36 \pm 0.08$ & $-0.74 \pm 0.13$ \\
\hline FDF-5058 & 010605.72 & -254626.3 & 23.48 & 21.07 & 2.027 & $-1.79 \pm 0.07$ & $+0.84 \pm 0.11$ \\
\hline FDF-5072 & 010605.76 & -254518.8 & 22.88 & 20.13 & 1.389 & $-1.50 \pm 0.06$ & $-0.42 \pm 0.08$ \\
\hline FDF-5135 & 010605.97 & -254443.8 & 23.89 & 20.76 & 2.346 & $-1.37 \pm 0.34$ & $+0.04 \pm 0.34$ \\
\hline FDF-5152 & 010606.01 & -254540.4 & 22.89 & 20.22 & 1.370 & $-1.32 \pm 0.05$ & $-0.28 \pm 0.06$ \\
\hline
\end{tabular}


Table 1. continued.

\begin{tabular}{|c|c|c|c|c|c|c|c|}
\hline ID & RA & Dec & $R$ & $K_{\mathrm{s}}$ & $z$ & $\beta_{\mathrm{b}}$ & $\gamma_{34}$ \\
\hline FDF-5165 & 010606.06 & -254443.4 & 23.53 & 20.39 & 2.346 & $-2.20 \pm 0.26$ & $-0.14 \pm 0.37$ \\
\hline FDF-5182 & 010606.13 & -254533.7 & 23.83 & 21.52 & 1.369 & $-1.78 \pm 0.09$ & $-0.39 \pm 0.10$ \\
\hline FDF-5190 & 010606.15 & -254443.1 & 24.66 & 22.78 & 2.347 & $-1.36 \pm 0.41$ & $-1.53 \pm 0.38$ \\
\hline FDF-5227 & 010606.29 & -254351.8 & 24.09 & 21.75 & 2.399 & $-1.34 \pm 0.32$ & 0.36 \\
\hline FDF-5236 & 010606.34 & -254542.2 & 22.54 & 18.94 & 1.153 & $-1.69 \pm 0.15$ & $-1.35 \pm 0.18$ \\
\hline FDF-5458 & 010607.18 & -254644.2 & 23.52 & 20.35 & 1.513 & $-1.30 \pm 0.07$ & $-1.00 \pm 0.13$ \\
\hline FDF-5585 & 010607.65 & -254550.6 & 21.78 & 17.79 & 1.074 & $+9.99 \pm 9.99$ & $-1.37 \pm 0.52$ \\
\hline FDF-5667 & 010607.94 & -254637.3 & 23.69 & 20.61 & 1.024 & $+9.99 \pm 9.99$ & $-1.36 \pm 1.59$ \\
\hline FDF-6024 & 010609.23 & -254814.1 & 22.27 & 19.85 & 2.372 & $-2.04 \pm 0.17$ & $-1.28 \pm 0.36$ \\
\hline FDF-6101 & 010609.50 & -254550.0 & 22.96 & 20.14 & 1.142 & $-0.88 \pm 0.13$ & $-2.34 \pm 0.18$ \\
\hline FDF-6344 & 010610.36 & -254333.4 & 23.32 & 22.18 & 1.663 & $-1.88 \pm 0.06$ & $-0.81 \pm 0.10$ \\
\hline FDF-6358 & 010610.39 & -254430.8 & 23.19 & 19.77 & 1.315 & $-0.64 \pm 0.18$ & $-0.32 \pm 0.21$ \\
\hline FDF-6372 & 010610.45 & -254829.0 & 23.55 & 20.86 & 2.349 & $-1.73 \pm 0.22$ & $+0.14 \pm 0.19$ \\
\hline FDF-6384 & 010610.49 & -254513.0 & 23.18 & 20.69 & 1.314 & $-1.08 \pm 0.07$ & $+0.71 \pm 0.08$ \\
\hline FDF-6407 & 010610.57 & -254531.7 & 23.95 & 20.70 & 2.162 & $-1.36 \pm 0.12$ & $+1.62 \pm 0.32$ \\
\hline FDF-6432 & 010610.65 & -254609.2 & 23.70 & 19.71 & 1.153 & $-0.11 \pm 0.27$ & $-1.04 \pm 0.34$ \\
\hline FDF-6547 & 010611.06 & -254649.1 & 23.74 & 21.27 & 1.212 & $-1.87 \pm 0.08$ & $-0.42 \pm 0.09$ \\
\hline FDF-6864 & 010612.19 & -254356.2 & 23.61 & 20.93 & 1.394 & $-1.57 \pm 0.06$ & $-0.34 \pm 0.08$ \\
\hline FDF-6934 & 010612.36 & -254456.8 & 23.08 & 20.16 & 2.445 & $-2.05 \pm 0.26$ & $-0.09 \pm 0.92$ \\
\hline FDF-6947 & 010612.40 & -254814.8 & 24.13 & 20.57 & 2.357 & $-1.87 \pm 0.16$ & $-3.46 \pm 0.19$ \\
\hline FDF-7029 & 010612.67 & -254558.4 & 23.86 & 21.52 & 2.374 & $-1.91 \pm 0.21$ & $-2.26 \pm 0.27$ \\
\hline FDF-7078 & 010612.80 & -254600.5 & 24.15 & 21.97 & 2.378 & $-1.47 \pm 0.35$ & $+2.70 \pm 0.98$ \\
\hline FDF-7223 & 010613.30 & -254432.7 & 23.27 & 20.12 & 1.051 & $+9.99 \pm 9.99$ & $-3.69 \pm 2.20$ \\
\hline FDF-7307 & 010613.60 & -254725.1 & 24.28 & 21.06 & 2.438 & $-2.00 \pm 0.33$ & $+2.19 \pm 0.86$ \\
\hline FDF-7342 & 010613.70 & -254613.2 & 24.07 & 20.70 & 2.375 & $-1.55 \pm 0.17$ & $-4.14 \pm 0.79$ \\
\hline FDF-7345 & 010613.71 & -254623.6 & 23.62 & 20.78 & 1.025 & $-1.95 \pm 0.08$ & $-1.11 \pm 0.11$ \\
\hline FDF-8604 & 010618.61 & -254817.5 & 21.53 & 18.04 & 1.142 & $-0.03 \pm 0.17$ & $-5.74 \pm 0.22$ \\
\hline Q0055-0017 & 005758.22 & -264059.2 & 23.84 & 19.68 & 1.128 & $-0.91 \pm 0.13$ & $-4.17 \pm 0.31$ \\
\hline Q0055-0023 & 005756.80 & -264104.7 & 23.06 & 19.87 & 1.436 & $-1.04 \pm 0.12$ & $-2.07 \pm 0.15$ \\
\hline Q0055-0024 & 005754.72 & -264107.5 & 22.33 & 19.94 & 1.374 & $-1.19 \pm 0.04$ & $-0.06 \pm 0.08$ \\
\hline Q0055-0050 & 005805.67 & -264127.0 & 23.66 & 19.25 & 1.002 & $+9.99 \pm 9.99$ & $-5.74 \pm 0.59$ \\
\hline Q0055-0052 & 005807.03 & -264127.0 & 21.88 & 18.99 & 2.175 & $-0.67 \pm 0.31$ & $-0.49 \pm 0.34$ \\
\hline Q0055-0119 & 005800.34 & -264226.5 & 23.20 & 19.37 & 1.059 & $-0.11 \pm 0.13$ & $-3.27 \pm 0.20$ \\
\hline Q0055-0131 & 005759.39 & -264237.2 & 22.93 & 19.42 & 1.367 & $-0.94 \pm$ & -0.5 \\
\hline Q0055-0157 & 005807.79 & -264252.9 & 22.33 & 19.91 & 1.314 & $+9.99 \pm 9.99$ & $+1.21 \pm 0.05$ \\
\hline Q0055-0255 & 005750.82 & -264352.5 & 23.47 & 18.75 & 1.105 & $+9.99 \pm 9.99$ & $-0.62 \pm 0.27$ \\
\hline Q0055-0262 & 005808.42 & -264355.4 & 23.30 & 19.45 & 1.075 & $+9.99 \pm 9.99$ & $+0.15 \pm 0.22$ \\
\hline SA02-1417 & 020933.32 & -043731.2 & 23.92 & 19.39 & 1.599 & $+9.99 \pm 9.99$ & $-0.69 \pm 0.32$ \\
\hline SA12-6301 & 120518.36 & -072343.3 & 22.70 & 19.49 & 1.760 & $+9.99 \pm 9.99$ & $-1.17 \pm 0.10$ \\
\hline SA12-6339 & 120532.70 & -072337.7 & 23.71 & 20.15 & 2.293 & $+9.99 \pm 9.99$ & $+0.96 \pm 0.12$ \\
\hline SA12-7250 & 120532.88 & -072232.8 & 23.98 & 19.94 & 1.900 & $+9.99 \pm 9.99$ & $+0.19 \pm 0.24$ \\
\hline SA15-4762 & 152353.27 & -000642.0 & 24.39 & 19.86 & 1.598 & $+9.99 \pm 9.99$ & $-7.11 \pm 0.37$ \\
\hline SA15-6396 & 152341.04 & -000510.8 & 23.92 & 21.00 & 1.928 & $+9.99 \pm 9.99$ & $+1.12 \pm 0.09$ \\
\hline SA15-6488 & 152345.55 & -000505.2 & 24.13 & 21.00 & 2.044 & $+9.99 \pm 9.99$ & $+1.01 \pm 0.22$ \\
\hline SA15-7353 & 152340.43 & -000354.1 & 24.68 & 19.89 & 2.091 & $+9.99 \pm 9.99$ & $+0.06 \pm 0.25$ \\
\hline
\end{tabular}

